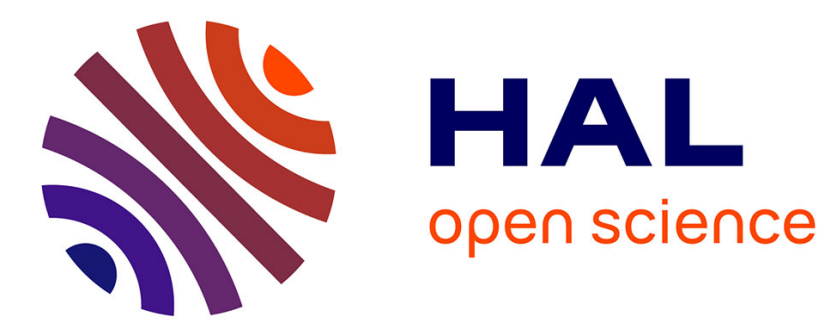

\title{
Perpendicular electric field in two-dimensional electron phase-holes: A parameter study
}

Q.M. Lu, Bertrand Lembège, J.B. Tao, S. Wang

\section{To cite this version:}

Q.M. Lu, Bertrand Lembège, J.B. Tao, S. Wang. Perpendicular electric field in two-dimensional electron phase-holes: A parameter study. Journal of Geophysical Research Space Physics, 2008, 113 (A11), pp.A11219. 10.1029/2008JA013693 . hal-00341539

\section{HAL Id: hal-00341539 \\ https://hal.science/hal-00341539}

Submitted on 7 Feb 2016

HAL is a multi-disciplinary open access archive for the deposit and dissemination of scientific research documents, whether they are published or not. The documents may come from teaching and research institutions in France or abroad, or from public or private research centers.
L'archive ouverte pluridisciplinaire HAL, est destinée au dépôt et à la diffusion de documents scientifiques de niveau recherche, publiés ou non, émanant des établissements d'enseignement et de recherche français ou étrangers, des laboratoires publics ou privés. 


\title{
Perpendicular electric field in two-dimensional electron phase-holes: A parameter study
}

\author{
Q. M. Lu, ${ }^{1,2}$ B. Lembege, ${ }^{3}$ J. B. Tao, ${ }^{1}$ and S. Wang ${ }^{1}$ \\ Received 20 August 2008; accepted 26 September 2008; published 25 November 2008.
}

[1] Previous multi-dimensional particle simulations have shown that electron phase-holes can be formed during the nonlinear evolution of bi-stream instability. In these holes, the parallel cut of the parallel electric field $\left(E_{\|}\right)$has bipolar structures while the parallel cut of the perpendicular electric field $\left(E_{\perp}\right)$ has unipolar structures. In this paper, two-dimensional (2D) electrostatic particle-in-cell simulations are performed to investigate the evolution of $E_{\perp}$ in such electron holes for different plasma conditions, and the generation mechanism of the unipolar structures of $E_{\perp}$ is also discussed. The electrons trapped in electron holes bounce in the parallel direction, which leads to transverse instability (Muschietti et al., 2000). At the same time, they gyrate in the background magnetic field, which tends to stabilize electron holes. In this way, the trapped electrons are forced to accumulate locally, and the charge density has variations along the perpendicular direction inside the electron holes. The balance between these two effects leads to the following results: in weakly magnetized plasma $\left(\Omega_{e}<\omega_{p e}\right.$, but $\Omega_{e}$ is comparable to $\omega_{p e}$. Where $\Omega_{e}$ and $\omega_{p e}$ are the electron cyclotron frequency and electron plasma frequency, respectively), electron holes have two-dimensional structures (isolated along both the parallel and perpendicular directions). Within such holes the parallel cut of $E_{\perp}$ has unipolar structures. In strongly magnetized plasma $\left(\Omega_{e}>\omega_{p e}\right)$, electron holes have one-dimensional structures along the direction perpendicular to the background magnetic field within which a series of islands (with alternate positive and negative $E_{\perp}$ ) develop because of the variations of the charge density along the perpendicular direction. Therefore one recovers that a parallel cut of $E_{\perp}$ has unipolar structures at the location of the holes. Present results show that the unipolar structure of $E_{\perp}$ in electron holes is attributed to the balance between the electron transverse instability and the stabilization of the background magnetic field. The unipolar structures of $E_{\perp}$ in electron holes last for hundreds to thousands of electron plasma periods. They are destroyed and the streaked structures are formed in the whole simulation domain after the electrostatic whistler waves are excited and have sufficiently large amplitude. The influences of the initial perpendicular thermal velocity of electrons (via temperature anisotropy) and the drift speed of electron beam on the structures of $E_{\perp}$ are also analyzed in details. At last, the relevance between our simulation results and the unipolar structures of the parallel cut of $E_{\perp}$ observed in the auroral region is discussed.

Citation: Lu, Q. M., B. Lembege, J. B. Tao, and S. Wang (2008), Perpendicular electric field in two-dimensional electron phaseholes: A parameter study, J. Geophys. Res., 113, A11219, doi:10.1029/2008JA013693.

\section{Introduction}

[2] Electrostatic solitary waves (ESWs), which move along the background magnetic field, have been observed

\footnotetext{
${ }^{1}$ CAS Key Laboratory of Basic Plasma Physics, School of Earth and Space Sciences, University of Science and Technology of China, Hefei, Anhui, China.

${ }^{2}$ State Key Laboratory of Space Weather, Chinese Academy of Sciences, Beijing, China.

${ }^{3}$ Centre d'étude des Environnements Terrestre et Planétaires, CNRS Université de Versailles-Saint Quentin, Vélizy, France.

Copyright 2008 by the American Geophysical Union. 0148-0227/08/2008JA013693
}

in different space environments, such as the magnetotail [Matsumoto et al., 1994], the auroral region [Ergun et al., 1998a; Cattell et al., 2002; Franz et al., 1998], the Earth's foreshock region [Bale et al., 1998], the magnetosheath [Pickett et al., 2004] and the solar wind [Mangeney et al., 1999]. They are positive potential pulses and the parallel cut of the parallel electric field $\left(E_{\|}\right)$has bipolar structures. These structures are modeled as electron phase-space holes which are stationary Bernstein-Greene-Kruskal (BGK) solutions of the Vlasov and Poisson equations [Bernstein et al., 1957; Krasovsky et al., 1997; Muschietti et al., 1999; Chen et al., 2005; $\mathrm{Ng}$ and Bhattacharjee, 2005]. Onedimensional (1D) particle simulations have shown that such electron holes can be formed through nonlinear evolution of 
electron bi-stream/beam instability [Omura et al., 1994, 1996; Mottez et al., 1997; Lu et al., 2005a, 2005b]. In the nonlinear evolution of the instability, the excited electric waves coalesce each other, and at last one or several solitary structures with bipolar electric field are formed. The electron phase-space holes are associated with these structures.

[3] The characteristics of electron phase-space holes in higher dimensions are a subject of ongoing study. Twodimensional (2D) and three-dimensional (3D) particle simulations of electron bi-stream instability have confirmed the formation of the electron phase-space holes and the associated bipolar structures of the parallel cut of $E_{\|}$during its nonlinear evolution, and these electron holes can emit electrostatic whistler waves in strongly magnetized plasma [Goldman et al., 1999; Oppenheim et al., 1999, 2001]. Recently, the parallel cut of the perpendicular electric field $\left(E_{\perp}\right)$ in the electron holes are observed to have unipolar structures by Polar and Fast spacecraft in the auroral region [Ergun et al., 1998a, 1998b; Franz et al., 1998, 2000, 2005; Grabbe and Menietti, 2006], and the generation mechanism for such structures of $E_{\perp}$ are received more and more attentions. Miyake et al. [2000] and Umeda et al. [2002] attributed such structures of $E_{\perp}$ to ion dynamics. In their 2-D particle simulations, they chose $\omega_{p e} \sim \Omega_{e}>\omega_{b}$ (where $\Omega_{e}$, $\omega_{p e}$ and $\omega_{b}$ are respectively the electron cyclotron frequency, the electron plasma frequency, and the bounce frequency of electrons trapped in electron holes), and a reduced ion-toelectron mass ratio $m_{i} / m_{e}=100$ was used. They found that the quasi-perpendicular lower hybrid waves excited during the evolution of electron bi-stream instability can couple with the electron holes. These one-dimensional (1D) holes are highly modulated by ion dynamics, or even $2 \mathrm{D}$ electron holes (isolated structures in both the parallel and perpendicular directions) can be formed. The parallel cut of $E_{\perp}$ has unipolar structures in such electron holes.

[4] Recently, with 2D particle-in-cell simulations Umeda et al. [2006] studied the nonlinear evolution of bi-stream instability in different plasma conditions, and the immobile background ions were assumed in the simulations to exclude the effect of ion dynamics. 2D electron holes were found to be formed in weakly magnetized plasma $\left(\omega_{p e}>\Omega_{e}>\right.$ $\left.\omega_{b}\right)$, and their parallel cut of $E_{\perp}$ was implied to have unipolar structures. In strongly magnetized plasma $\left(\Omega_{e}>\omega_{p e}\right), 1 \mathrm{D}$ kinked electron holes (the perpendicular extension of the structures is infinite and the parallel extension is limited) may be formed during the nonlinear evolution of bi-stream instability, and the parallel cut of $E_{\perp}$ in such structures was also implied to have unipolar structures [Goldman et al., 1999; Oppenheim et al., 1999, 2001; Umeda et al., 2006]. Ion dynamics were considered to be inconsequential for the formation of such kinked electron holes. Therefore ion dynamics is not necessary for the formation of such unipolar structures. In this paper, a new generated mechanism, which is not based on ion dynamics, is proposed to explain the unipolar structures of the parallel cut of $E_{\perp}$ in electron holes. We attribute the formation of such structures to the balance between the transverse instability and the stabilization of the background magnetic field. The transverse instability in electron holes was firstly proposed by Muschietti et al. [2000], which is due to the motion of the electrons trapped in the holes and is a self-focusing type of instability. At the same time, the lifetime of the unipolar structures of the electron holes for different plasma conditions (such as the background magnetic field, electron beam drift velocity and the initial electron temperature anisotropy) is also investigated with the help of 2D electrostatic particle-in-cell (PIC) simulations. At last, the relevance between our simulation results and the unipolar structures of $E_{\perp}$ observed in the auroral region is discussed.

[5] The paper is organized as follows. Section 2 presents the simulation model and plasma conditions. Simulation results are described in section 3 . Section 4 summarizes the discussion and conclusions.

\section{Simulation Model}

[6] A 2D electrostatic PIC code with periodic boundary conditions is implemented in our simulations [Decyk, 1995; Lu and Cai, 2001]. The simulation system is taken in the $x-y$ plane with a uniform background magnetic field $\mathbf{B}_{0}$ along the $x$ direction. Two electron components are employed in the simulations, and their initial velocity distributions are assumed to be bi-Maxwellian. The initial bi-Maxwellian distribution is assumed to be

$$
f_{e}: \exp \left(-\frac{\left(v_{x}-V_{d x}\right)^{2}}{2 v_{T e}^{2}}-\frac{v_{y}^{2}+v_{z}^{2}}{2 v_{T e}^{2}} \frac{T_{\| e}}{T_{\perp e}}\right),
$$

where $v_{T e}\left(=\sqrt{k_{B} T_{\| e} / m_{e}}\right)$ is the initial parallel thermal velocity, $V_{d x}$ is the initial parallel mean velocity, $T_{\| e}$ and $T_{\perp e}$ are the initial parallel and perpendicular temperatures, respectively. These two electron components have equal density $\left(n_{e 1}=n_{e 2}=n_{e}\right)$, initial parallel and perpendicular temperatures $\left(T_{\| e 1}=T_{\| e 2}=T_{\| e}, T_{\perp e 1}=T_{\perp e 2}=T_{\perp e}\right)$. Initially, the first component has no drift velocity and the second has drift velocity $V_{d}$. A Maxwellian distribution of ions with a mean velocity of $V_{d x}=0$ is also introduced, and its temperature is the same as the parallel temperature of electrons.

[7] In the simulations, the densities and velocities are expressed in units of the total unperturbed density $n_{0}=n_{i}=$ $n_{e 1}+n_{e 2}=2 n_{e}$ (where $n_{i}$ is the ion number density) and initial parallel thermal velocity $v_{T e}$, respectively. Space and time are normalized to the electron Debye length $\lambda_{D}=$ $\left(\varepsilon_{0} k_{B} T_{\| e} / n_{0} e^{2}\right)$ and the inverse of the electron plasma frequency $\omega_{p e}=\left(n_{0} e^{2} / m_{e} \varepsilon_{0}\right)^{1 / 2}$. The electric field is normalized to $m_{e} \omega_{p e} v_{T e} / e$. Grid size units $\lambda_{D} \times \lambda_{D}$ are used in the simulations, and the time step is $0.02 \omega_{p e}^{-1}$. The average number of particles in every grid size for each component (background electrons, beam electrons and ions) is 100. The number of grids used in the simulations is $512 \times 256$. In order to identify the role of ion dynamics in the formation of the unipolar structures of the parallel cut of $E_{\perp}$ in electron holes, we firstly use the realistic ion to electron mass ratio $m_{i} / m_{e}=1836$, then compare the simulation results with different mass ratios $m_{i} / m_{e}=\infty$, and 100 . When we use $m_{i} /$ $m_{e}=\infty$, ions are actually immobile and their dynamics are excluded.

\section{Simulation Results}

[8] The evolution of electron bi-stream instability has been extensively studied by previous work [Goldman et 
Table 1. Summary of Simulations (Runs 1-9)

\begin{tabular}{ccccc}
\hline Run Number & $\Omega_{e} / \omega_{p e}$ & $V_{d} / v_{T e}$ & $T_{\perp e} / T_{\| e}$ & $m_{i} / m_{e}$ \\
\hline 1 & 0.4 & 4.0 & 1.0 & 1836 \\
2 & 2.0 & 4.0 & 1.0 & 1836 \\
3 & 10.0 & 4.0 & 1.0 & 1836 \\
4 & 0.4 & 4.0 & 3.0 & 1836 \\
5 & 2.0 & 4.0 & 3.0 & 1836 \\
6 & 0.4 & 4.5 & 1.0 & 1836 \\
7 & 2.0 & 4.5 & 1.0 & 1836 \\
8 & 2.0 & 4.0 & 1.0 & $\infty$ \\
9 & 2.0 & 4.0 & 1.0 & 100 \\
\hline
\end{tabular}

al., 1999; Oppenheim et al., 2001; Lu et al., 2005a]. During the linear growth stage, the excited waves are nearly monochromatic and have a substantial degree of coherence perpendicular to the background magnetic field, which leads to an ensemble of ridges and valleys elongated along the $y$ direction for the parallel electric field $\left(E_{x}\right)$. After the amplitude of $E_{x}$ becomes sufficiently large, a part of electrons is trapped by the waves and nonlinear kinetic effects develop. In this paper, our main interests are focused on the unipolar structures of $E_{\perp}$ in electron holes and their generated mechanism during the nonlinear evolution of the instability under different plasma conditions. We have performed a total of 9 numerical simulations. The key parameters are listed in Table 1. Runs 1-3 investigate the influences of the background magnetic field on the nonlinear evolution of electron bi-stream instability, Runs 4-5 consider the effects of the initial electron temperature anisotropy, Run 6-7 and Run 8-9 discuss the effects of electron beam drift velocity and ion-to-electron mass ratio, respectively. In the follows, we discuss these effects separately.

\subsection{Effects of the Background Magnetic Field}

[9] Figure 1 shows the simulation results for Run 1, which corresponds to weakly magnetized plasma. In the figure, the left column shows the time evolution of the electric field energies $E_{x}^{2}$ and $E_{y}^{2}$, which is normalized by $m_{e}^{2} \omega_{p e}^{2} v_{t e}^{2} / e^{2}$. The three right columns show the electric field components (a) $E_{x}$ and (b) $E_{y}$ at $\omega_{p e} t=320,1060$, and 2740 between $x=0$ and $x=200 \lambda_{D}$. With the excitation of the bi-stream instability, the electric field energy $E_{x}^{2}$ increases rapidly. When the amplitude of $E_{x}$ is sufficiently large, the electric field energy $E_{y}^{2}$ begins to grow because of the nonlinear effects. After the electric field energies $E_{x}^{2}$ and $E_{y}^{2}$ attain their maximum values (saturation), they begin to decrease because of waves coalesce [Goldman et al., 1999; Lu et al., 2005a]. From the electric field components $E_{x}$ and $E_{y}$ at $\omega_{p e} t=320$, we can find that the waves coalesce into several tubes elongate along the $y$ direction. At the same time, the tubes begin to break into several segments along $y$ direction. As a result, several two-dimensional solitary structures (and associated electron holes), which are isolated in both the parallel and perpendicular directions, are formed around $\omega_{p e} t=500$. The electric field components $E_{x}$ and $E_{y}$ at $\omega_{p e} t=1060$ of Figure 1 clearly shows several such electron holes. If we cut the edges of the electron holes in the parallel direction, for example along $y=96 \lambda_{D}$, we can find that in the electron hole the parallel electric field has bipolar structures and the perpendicular electric field has unipolar structures. As time goes on, such two-dimensional electron holes become weaker and weaker, until at about $\omega_{p e} t=3000$ they are too weak to be observed.

[10] Figures 2 and 3 describe respectively the simulation results for Run 2 and 3, which correspond to strongly magnetized plasma. Compared with weakly magnetized plasma, both $E_{x}^{2}$ and $E_{y}^{2}$ field energy become larger in strongly magnetized plasma although their time evolutions are qualitatively similar. As in weakly magnetized plasma, one or several electron holes can be formed through wave coalesce, and but takes place at earlier times (around $\omega_{p e} t=300$ and 260 for Run 2 and 3, respectively). In contrast with weakly magnetized plasma case, the electron holes in strongly magnetized plasma are mainly one-dimensional (the extension along the $y$ direction is infinite), and they can persist without breaking into segments until to the end in our simulations. Within these one-dimensional electron holes, a series of islands (with alternate positive and negative $E_{y}$ ) develops along the direction perpendicular to the background magnetic field, which can last for about two thousand of plasma periods. Therefore, in strongly magnetized plasma, bipolar and unipolar structures are recovered for the parallel cut of $E_{x}$ and $E_{y}$, respectively, which now are associated with one-dimensional electron holes. These are similar to the structures observed in weakly magnetized plasma (associated with two-dimensional electron holes shown in Figure 1). Such bipolar and unipolar profiles are easily identified in Figure 4, which shows the parallel cut of $E_{x}$ and $E_{y}$ along (a) $y=25 \lambda_{D}$ and (b) $y=95 \lambda_{D}$ (as illustrated by the dashed lines in Figure 2) at $\omega_{p e} t=640$ for Run 2. The electron phase-space holes are unstable to electrostatic whistler waves in strongly magnetized plasma, as described by Goldman et al. [1999]. The electrostatic whistler waves are generalized Langmuir waves, which propagate oblique to the background magnetic field. The electrostatic whistler waves are considered to be emitted by periodic motions of trapped electrons in the potential structures of the electron hole [Berthomier et al., 2002] or vibrations of kinked tubes [Newman et al., 2001]. The electrostatic whistler waves begin to be excited at about $\omega_{p e} t=1200$ and $\omega_{p e} t=1100$ for Run 2 and 3, respectively. At about $\omega_{p e} t=2000$, the unipolar $E_{y}$ structures are destroyed for both Run 2 and 3, and they are replaced by streaked structures in the simulation domain.

[11] The unipolar $E_{y}$ structures in electron holes (or variations of $E_{y}$ in electron holes) can be explained by the variations of the charge density along the y direction in the corresponding electron holes. The variations of the charge density in electron holes are shown in Figure 5, which shows the charge density $\rho$ normalized by $n_{0} e$ for (a) Run 1 at $\omega_{p e} t=1060$, (b) Run 2 at $\omega_{p e} t=640$, and (c) Run 3 at $\omega_{p e} t=640$. In general, the charge density is positive in the electron holes, which also has two-dimensional structures in Run 1. In Run 2 and Run 3, the charge density has onedimensional structures, but still has variations along the y direction same as $E_{y}$. This can be seen more clearly in Figure 6 . Figure 6 shows the charge density $\rho, \partial E_{x} / \partial x, \partial E_{y} / \partial y$ and $E_{y}$ along the $y$ direction inside the electron hole for (a) Run 2 at $\omega_{p e} t=640$ around $x=105 \lambda_{D}$, and (b) Run 3 at $\omega_{p e} t=640$ around $x=110 \lambda_{D}$. The dash dot lines denote the average charge density $\rho_{0}$ in the electron hole, and it is a positive value. The average charge density $\rho_{0}$ roughly stands for the contributions of $\partial E_{x} / \partial x$ in the electron hole. 

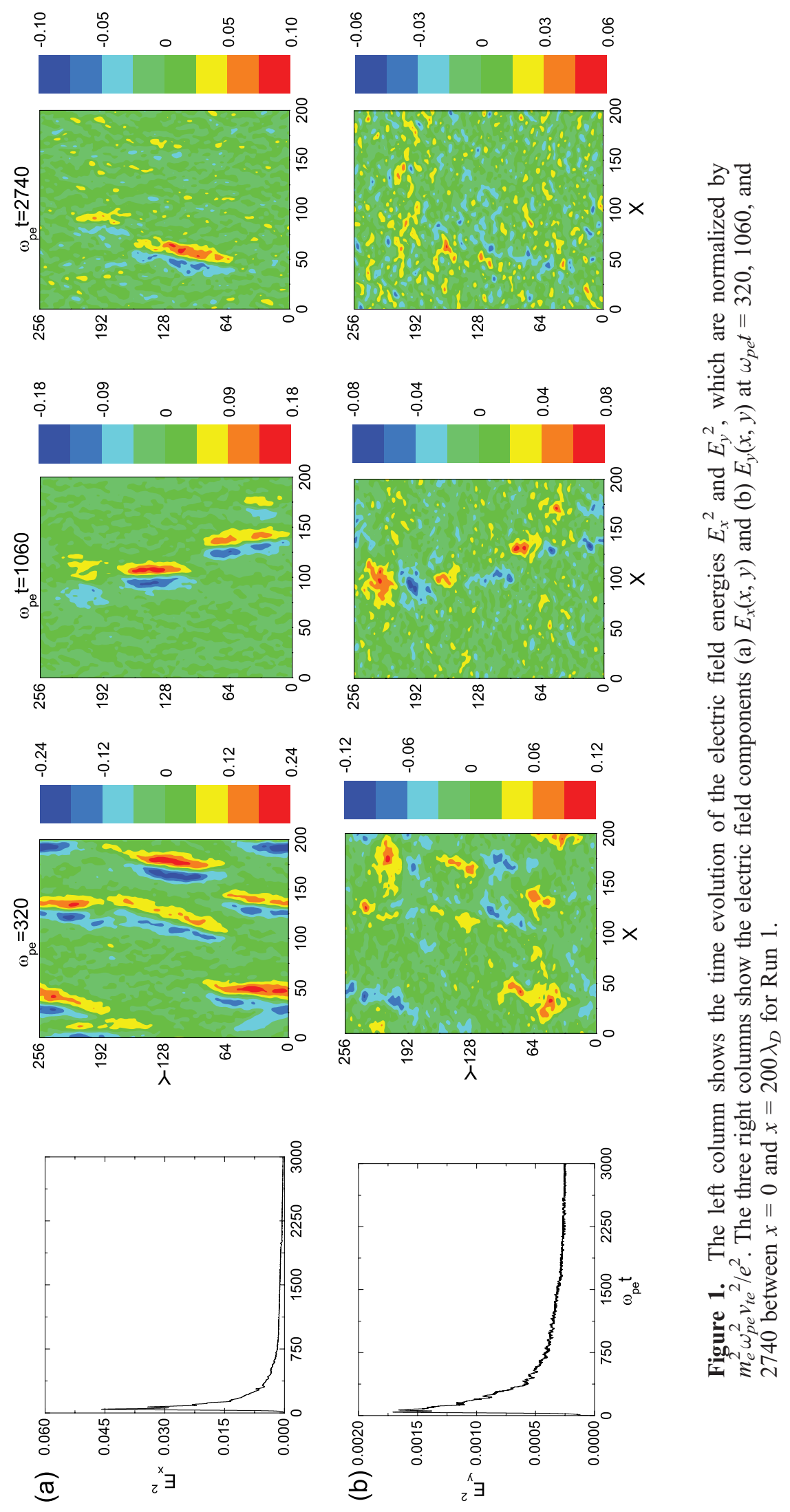

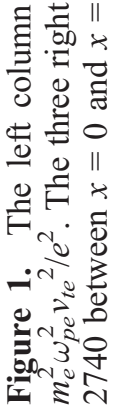



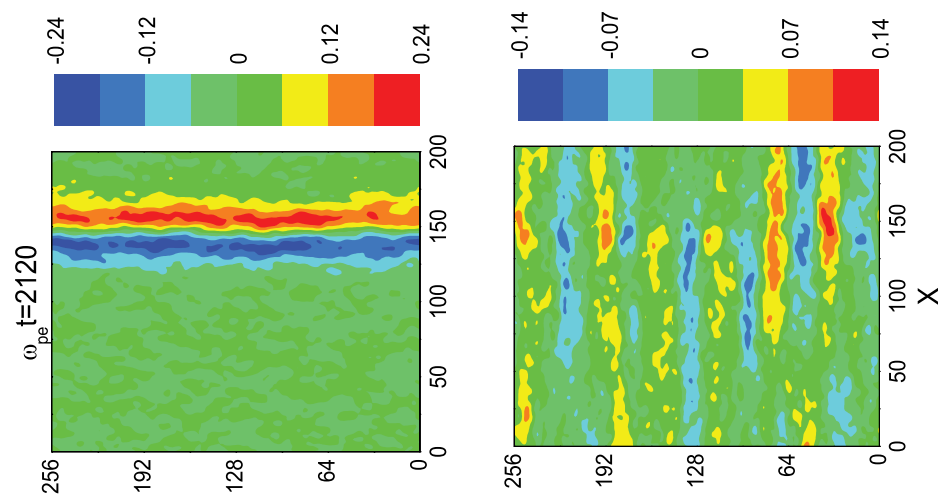

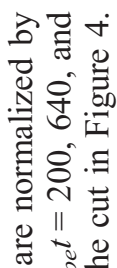
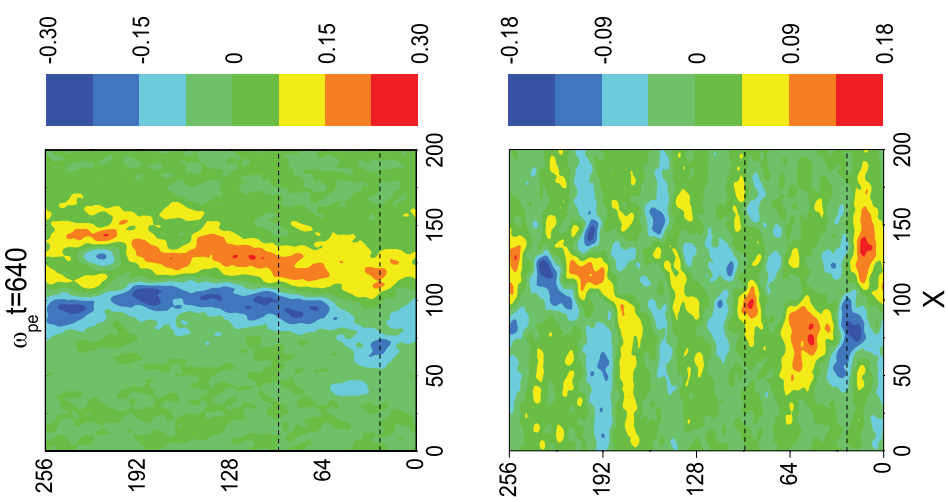

들

$3 \overbrace{}^{2} \mathscr{0}$

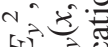

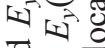

氙

过葛

$\mathscr{2}$ ว $\frac{0}{*}$

क्षे

可处.

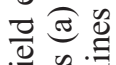

结告

记
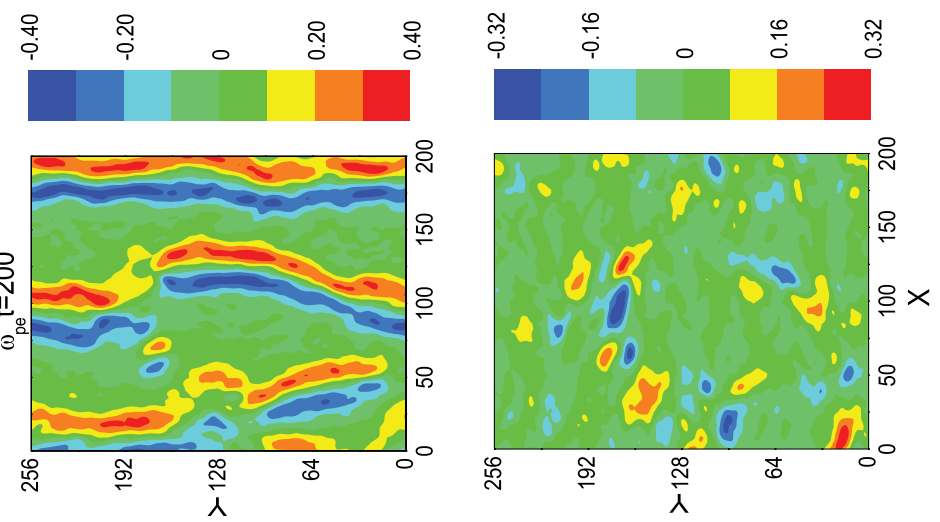

픙

들

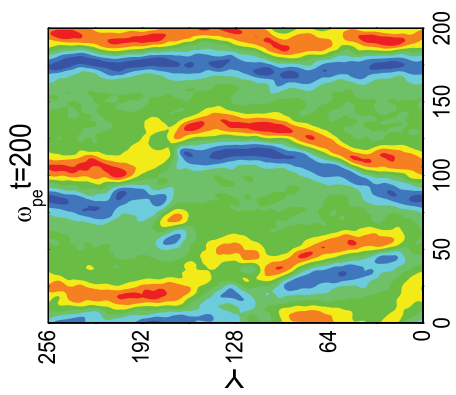

을

긍 $\frac{0}{0}=$

d)

常毛

을

क

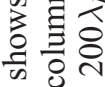

音泀
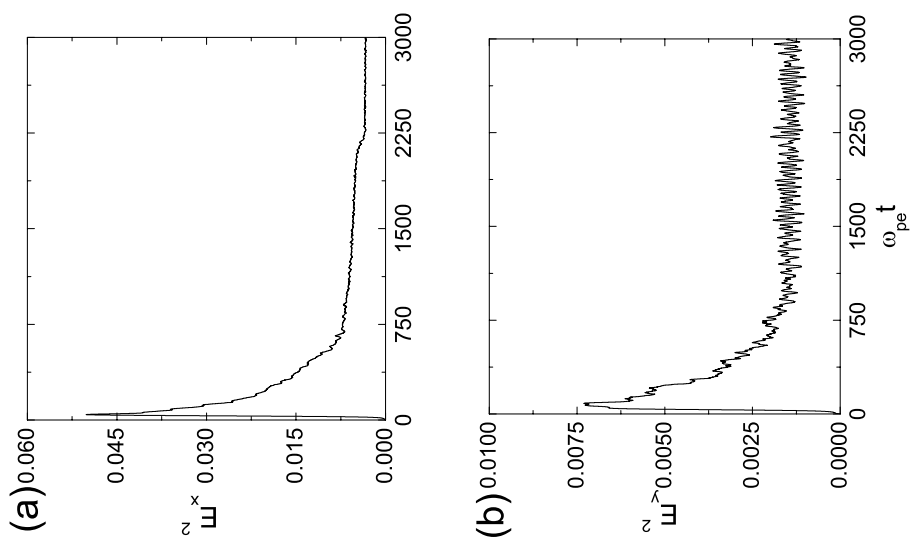

必 o EЕ ก $\frac{0}{2}$

근? 인 30

沿 

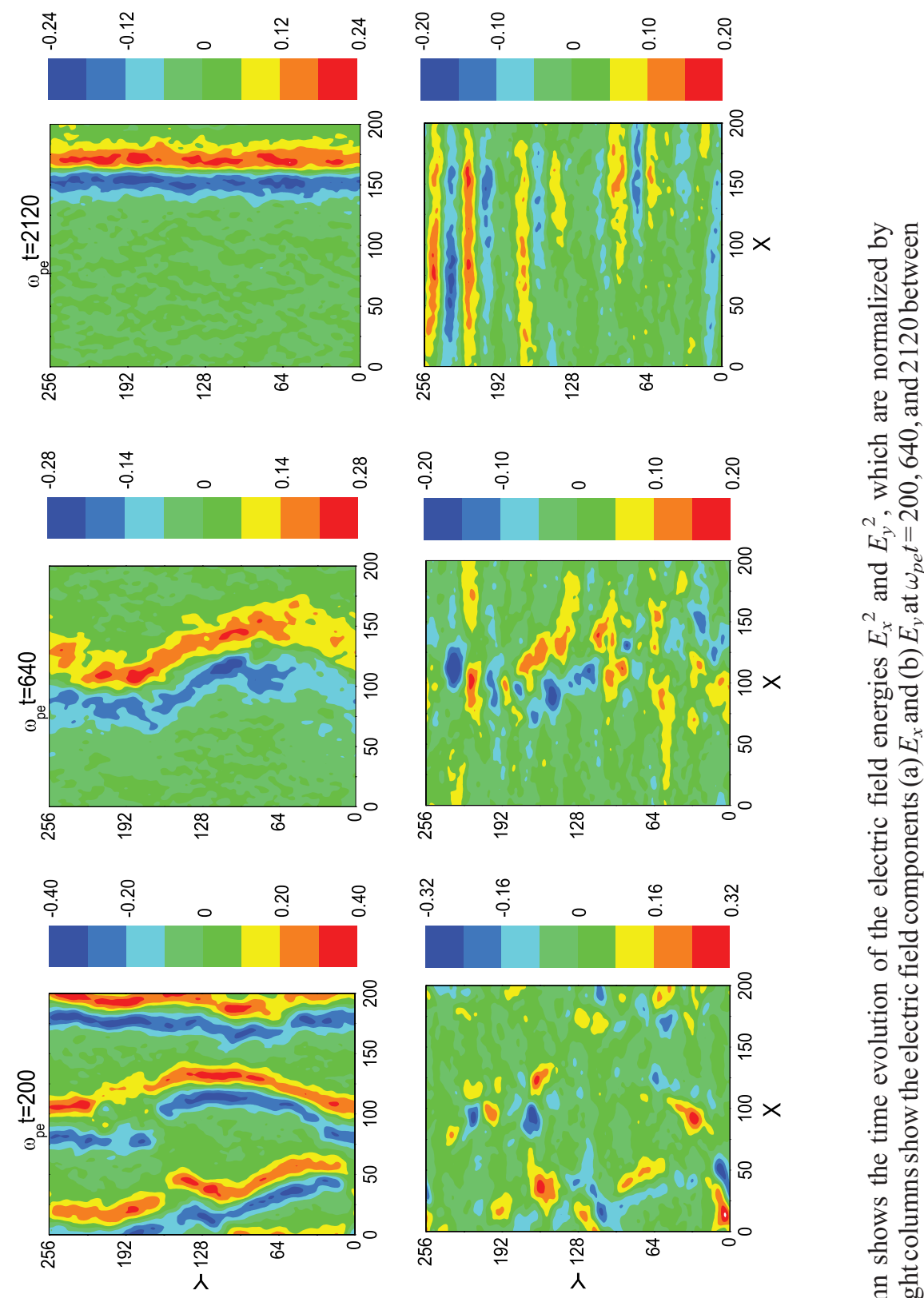

웡

$\mathrm{Nan}^{\circ} \mathrm{N}$ 너휴 त $\frac{\pi}{2}$ 过武 की
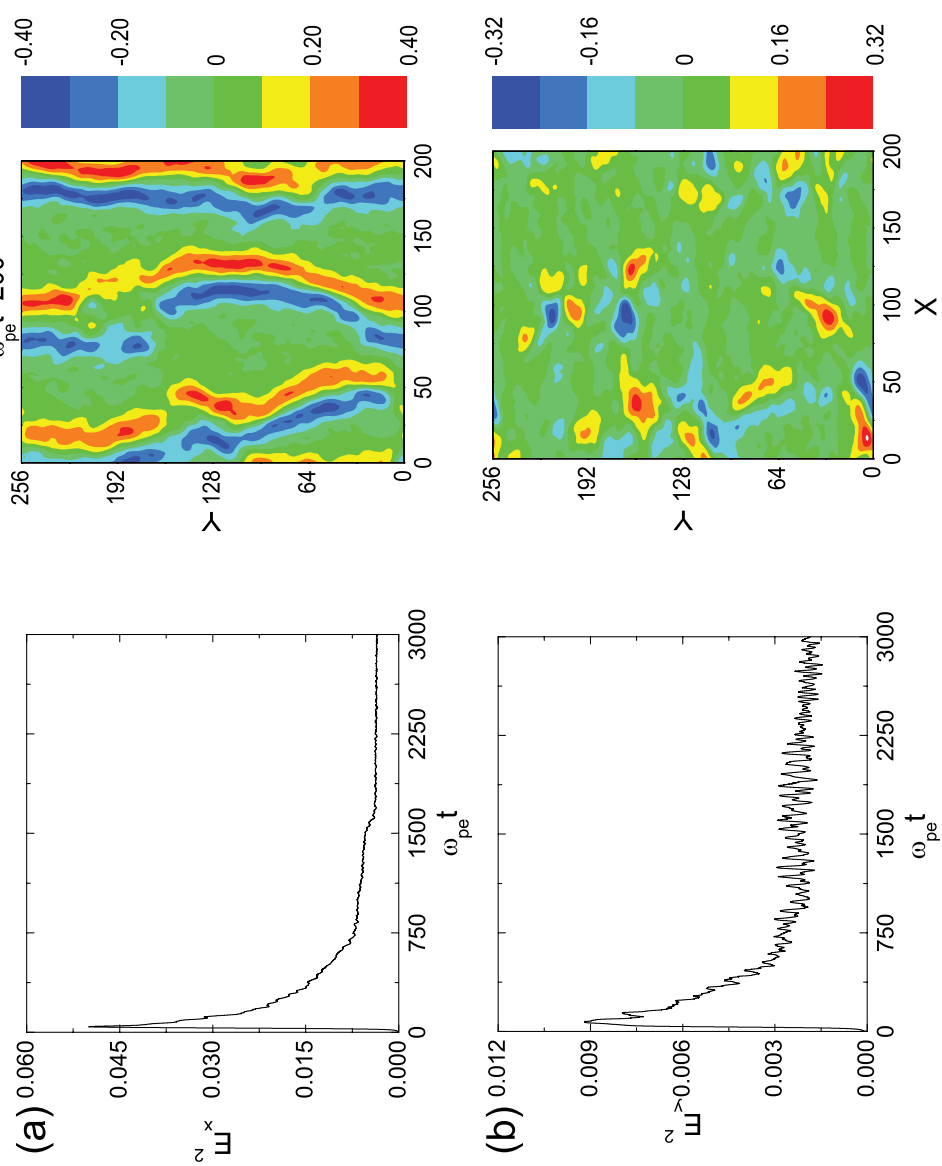

응

40

을

잉

वे

.

क क

吃先

क

晋全

8 巳

可

हैं

त.

${ }^{2}{ }^{2}=$

些 

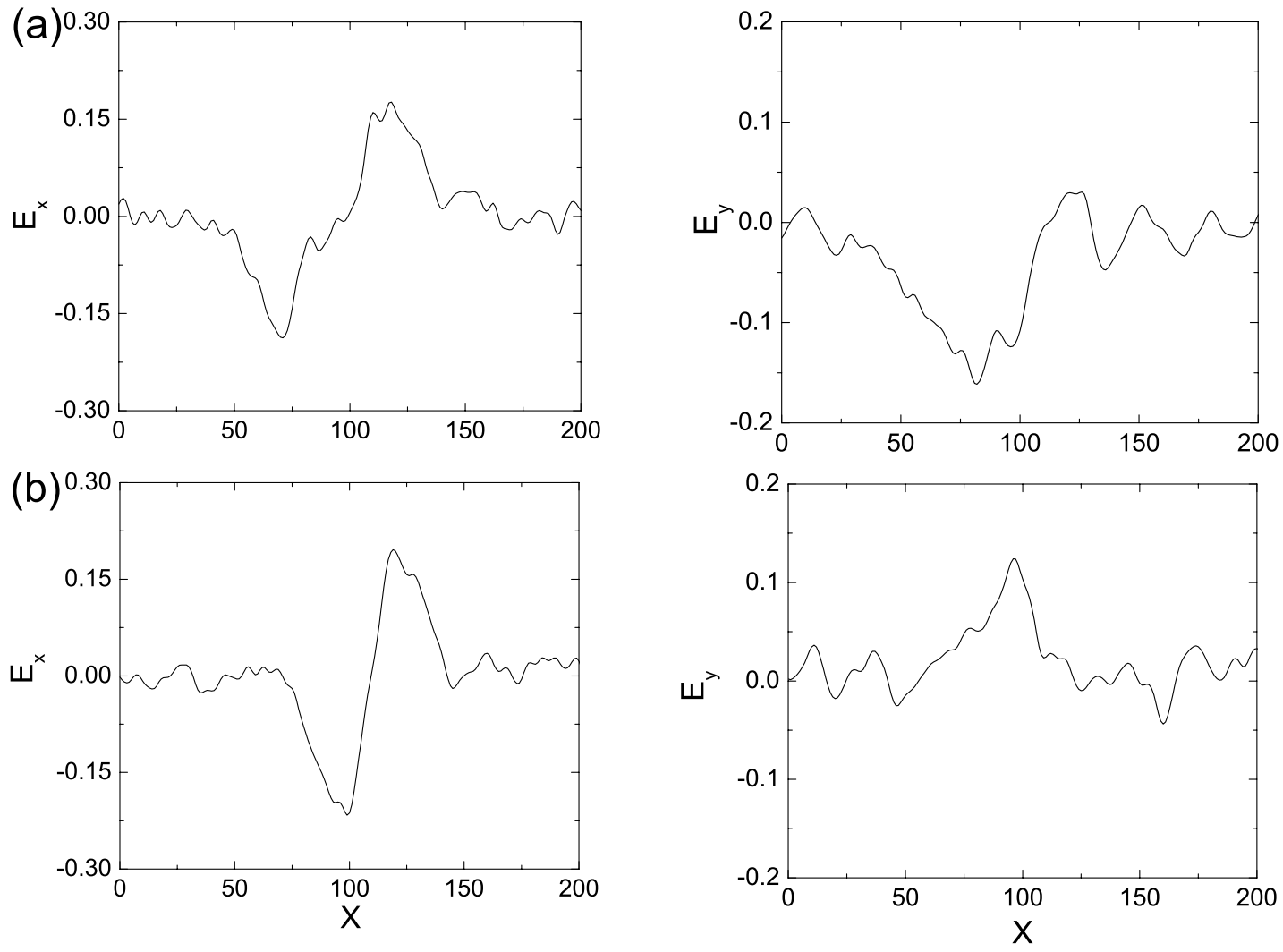

Figure 4. The parallel cut of the electric field $E_{x}(x)$ and $E_{y}(x)$ along (a) $y=25 \lambda_{D}$ and (b) $y=95 \lambda_{D}$ (as indicated by the dashed lines in Figure 2) at $\omega_{p e} t=640$ for Run 2.

While $\rho$ and $\partial E_{y} / \partial y$ are positively correlated in the electron hole: $\partial E_{y} / \partial y$ increases or decreases when $\rho$ increases or decreases. Therefore the variations of $\rho$ along the $y$ direction lead to the corresponding variations of $\partial E_{y} / \partial y$ in the electron holes, and produce the regular structures of $E_{y}$ (with alternate positive and negative $E_{y}$ ) along the $y$ direction. Of course, there is an approximate $\pi / 2$ phase difference between $E_{y}$ and $\partial E_{y} / \partial y$ (or $\rho$ ) along the $y$ direction in the electron hole. This can be clearly found in the figure. Complementary analysis shows that the electron holes are moving along the $x$ direction, and its propagation speed is about $1 / 2 V_{d}$, which is $2.0 v_{t e}$ for Run 2 and 3 . Figure 7 represents $E_{y}$ component along the $y$ direction inside the electron hole at different times for (a) Run 2 and (b) Run 3. In the figure, we follow the same electron hole, which is propagating along the $x$ direction. The $E_{y}$ structures look almost stationary along the $y$ direction. Therefore the structures of $E_{y}$ move with the electron hole in the $x$ direction, and they don't propagate along the $y$ direction.

[12] The variations of $\rho$ along the $y$ direction in the electron holes are due to the local charge accumulation, which is the result of the nonlinear equilibrium between the transverse instability and the stabilization of the background magnetic field in the electron holes [Muschietti et al., 2000]. The transverse instability is a self-focusing type of instability acting at the level of the particle trajectories. When the bounce frequency of the trapped electrons in the electron holes $\omega_{b}$ is larger than their gyrofrequency $\Omega_{e}\left(\Omega_{e}<\omega_{b}\right)$, the electrons are focused by the transverse gradients of the potential into those area that already have a surplus of (a) Run $1 \quad \omega_{p e} t=1060$

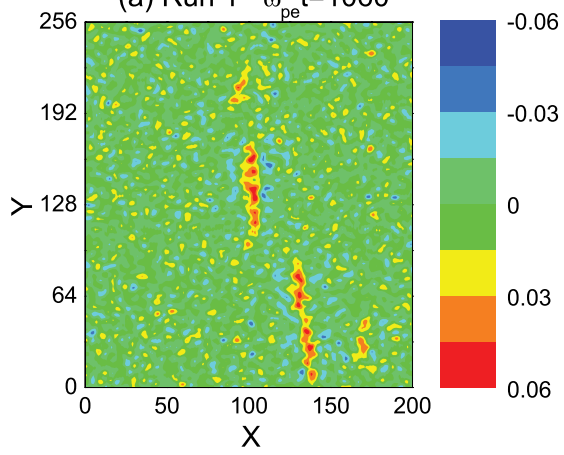

(b) Run $2 \omega_{p e} t=640$

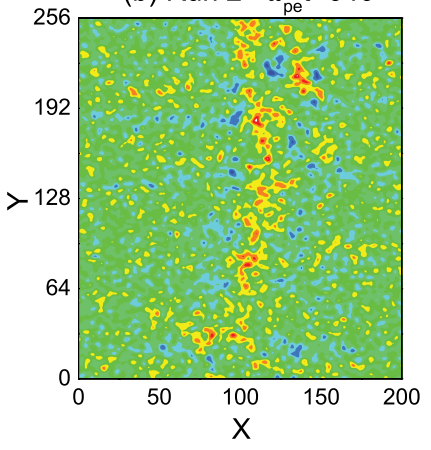

(c) Run $3 \omega_{p e} t=640$

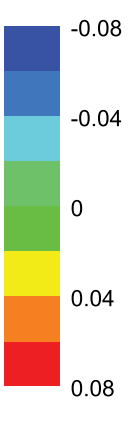

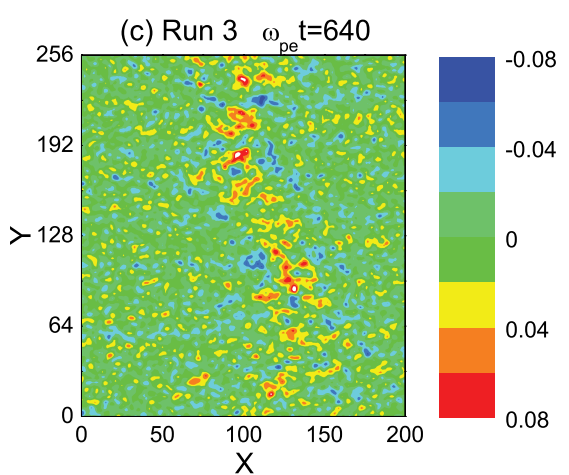

Figure 5. The charge density $\rho$ normalized by $n_{0} e$ for (a) Run 1 at $\omega_{p e} t=1060$, (b) Run 2 at $\omega_{p e} t=640$, and (c) Run 3 at $\omega_{p e} t=640$. 

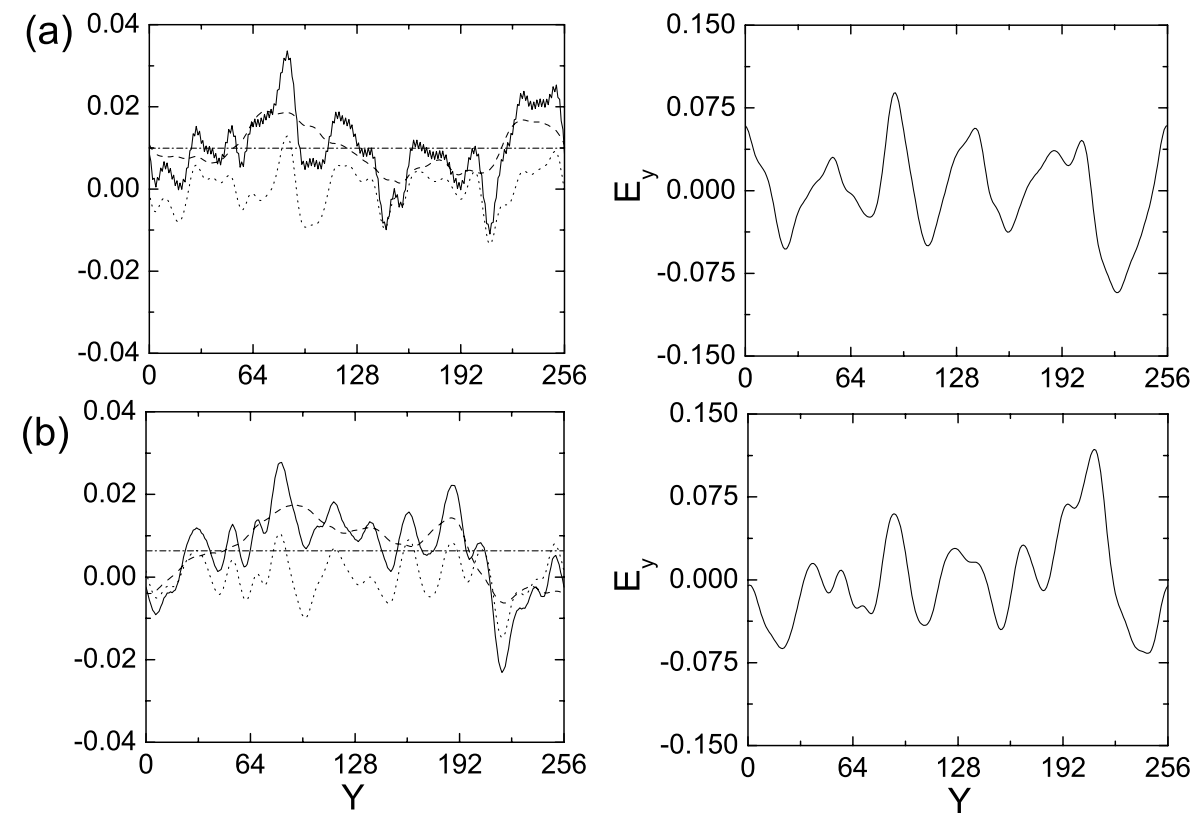

Figure 6. The charge density $\rho, \partial E_{x} / \partial x, \partial E_{y} / \partial y$ and $E_{y}$ along the $y$ direction inside the electron hole. (a) describes the results at $\omega_{p e} t=640$ for Run 2. The electron hole is located around $x=105 \lambda_{D}$. (b) describes the results at $\omega_{p e} t=640$ for Run 3. The electron hole is located around $x=110 \lambda_{D}$. Here $\rho$, $\partial E_{x} / \partial x, \partial E_{y} / \partial y$ and $E_{y}$ are the average values between $95 \lambda_{D}<x<114 \lambda_{D}$ and $100 \lambda_{D}<x<119 \lambda_{D}$ for Runs 2 and 3, respectively, and the noise is filtered by FFT. In the figure, the solid, dash, dot and dash dot line in the left column denote the $\rho, \partial E_{x} / \partial x, \partial E_{y} / \partial y$ and the average charge density $\rho_{0}$ in the electron hole, respectively.

electrons. This results in larger transverse gradients and thus the instability of the electron holes. With the increase of the background magnetic field, when $\Omega_{e}$ is larger than $\Omega_{e}\left(\Omega_{e}>\right.$ $\omega_{b}$ ) the magnetic field guides the trapped electrons bounce back and forth in electron holes. It can prevent the trapped electrons from being focusing by the transverse gradients of the potential, and a stable nonlinear equilibrium stage can be attained. The undulation of the charge density along the $y$ direction can be found at this equilibrium stage. Therefore, if $\Omega_{e}>\omega_{b}$, although the electron holes are one-dimensional (aligned along the $y$ direction), their perpendicular electric field $E_{y}$ has variations along the $y$ direction due to local charge accumulation. If we decrease the magnetic field such as $\Omega_{e}: \omega_{b}$, two-dimensional electron holes are formed. If we further decrease the magnetic field such as $\Omega_{e}<\omega_{b}$, there is no electron hole formed because of the transverse instability (not shown).

[13] The above conditions on the formation of $1 \mathrm{D}$ and 2D electron holes can be verified by following the trajectories of the trapped electrons. Figure 8 shows the time evolutions of $v_{x}^{\prime}=v_{x}-V_{E H}$ (where $V_{E H}$ is the propagation speed of the electron hole) and $v_{y}$ of a typical electron trajectory trapped in one electron hole for (a) Run 1 and (b) Run 2. The trajectory of the electron is calculated as follows: we put the (a)

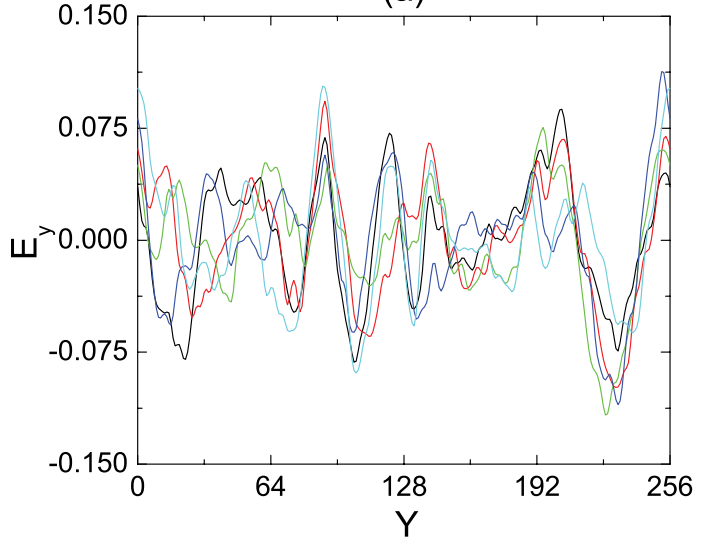

(b)

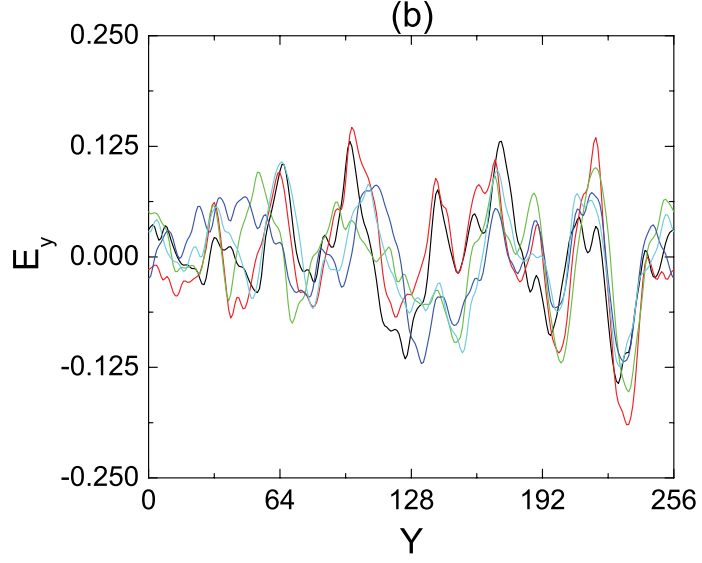

Figure 7. The electric field $E_{y}(y)$ along the $y$ direction measured within the electron hole at different times. The black, red, green, blue, and cyan colored lines correspond to $\omega_{p e} t=620,640,660,680$ and 700 from results of (a) Run 2 and (b) Run 3. Here the value of $E_{y}(y)$ is the average values near the center of the same electron hole ( 40 grids along $x$ direction), and the noise is filtered by FFT. 

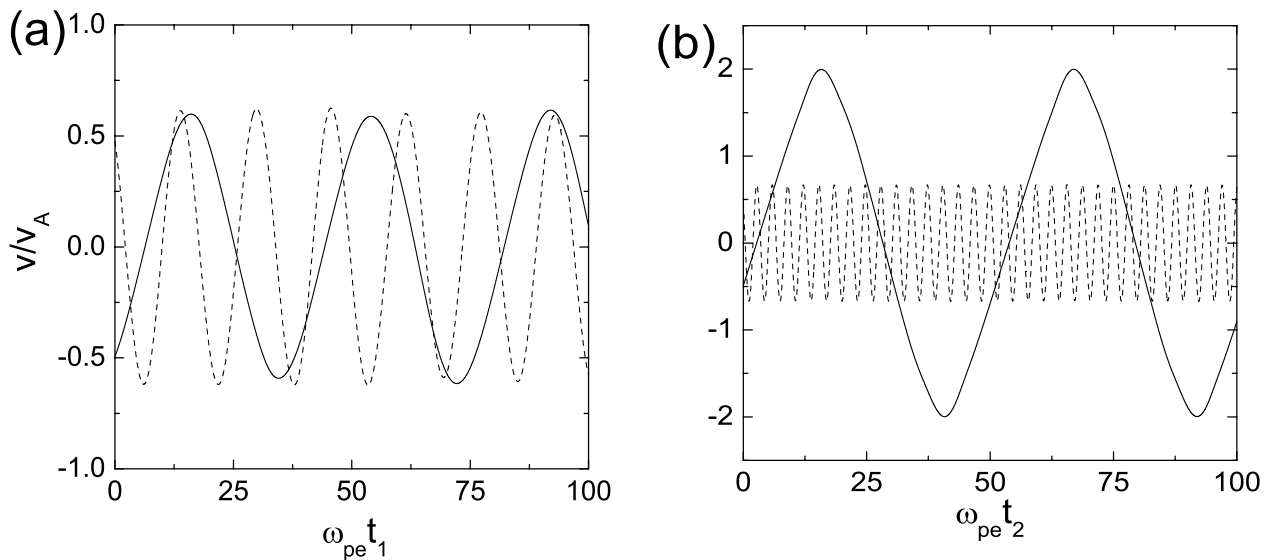

Figure 8. The time evolutions of $v_{x}^{\prime}=v_{x}-V_{E H}$ (where $V_{E H}$ is the propagation speed of the electron hole, and $v_{x}^{\prime}$ is the $x$ component of the electron velocity in the frame propagating with the speed of the electron hole) and $v_{y}$ of a typical electron trajectory trapped in one electron hole for (a) Run 1 and (b) Run 2. In the figure, the solid and dash lines denote $v_{x}^{\prime}$ and $v_{y}$, respectively.

particle into the electron hole at (a) $\omega_{p e} t=640$ for Run 1 and (b) $\omega_{p e} t=1060$ for Run 2, and then we follow the electron trajectory in electric and magnetic field fields. From the figure, we can find that the electron is trapped by the hole. The bounce frequency of the electron in the parallel direction $\left(\omega_{b}\right)$ is about $0.16 \omega_{p e}$ and $0.12 \omega_{p e}$ for Run 1 and Run 2, and its motion in the $y$ direction can be approximated as gyromotion in the background magnetic field with frequency of $\Omega_{e}$, which is $0.4 \omega_{p e}$ and $2.0 \omega_{p e}$ for Run 1 and Run 2, respectively. Therefore, in Run 2, the trapped electrons can lead to local charge accumulation along the $y$ direction with the variations of the charge density. In this way, although the electron holes are one-dimensional (aligned along the $y$ direction), their perpendicular electric field $E_{y}$ has variations along the $y$ direction. The similar results can be obtained in Run 3 (the $\omega_{b}$ and $\Omega_{e}$ are $0.12 \omega_{p e}$ and $10.0 \omega_{p e}$, respectively). If we decrease the magnetic field such that the gyro-frequency of the trapped electrons in the $y$ direction is comparable to the bounce frequency, twodimensional electron holes are formed (Run 1). If we further decrease the magnetic field to about $\Omega_{e} / \omega_{p e}=0.2$, there is no electron hole formed because of the transverse instability (not shown).

\subsection{Effects of the Initial Electron Temperature Anisotropy}

[14] As described by Muschietti et al. [2000], the increase of the electron perpendicular temperature can suppress the transverse instability in electron holes. Run 4 and 5 analyze the effects of an initial electron temperature anisotropy on the evolution of electron bi-stream instability. The results are shown in Figures 9 and 10, respectively, for weakly and strongly magnetized plasma conditions. For weakly magnetized plasma (Run 4), the transverse instability is suppressed by the larger electron perpendicular thermal velocity, and the electron holes are easily formed. At about $\omega_{p e} t=360$, several one-dimensional electron holes begin to be formed. From about $\omega_{p e} t=660$, the one-dimensional electron holes begin to break into several two-dimension holes associated with tubes breaking (Figure 9). At last, several two-dimensional holes are formed. In this case, the temperature anisotropy leads to a lower $E_{y}^{2}$ field energy at saturation time, but a slightly higher value in the late nonlinear stage of the bi-stream instability (to compare left column of Figures 1 and 9). In particular, the electric fields components have higher amplitudes at the electron hole locations (to compare Figures 1 and 9 at $\omega_{p e} t=2740$ ). This is in agreement with the fact that the initial anisotropy counteracts the resulting parallel diffusion of electrons which contributes to destroying the electron holes. However, in strongly magnetized plasma (Run 5), the electron temperature anisotropy has no obvious effects on the evolution of bi-stream instability. In both weakly and strongly magnetized plasma, the parallel cut of $E_{y}$ still has unipolar structures in electron holes.

\subsection{Effects of Electron Beam Drift Velocity}

[15] Runs 6 and 7 present the evolution of electron bistream instability when the drift velocity of beam electrons increases, and the results for weakly and strongly magnetized plasmas are shown in Figures 11 and 12, respectively. For weakly magnetized plasma (Run 6), the time evolution of the bi-stream instability is similar to results of Run 1, after the drift velocity of beam electrons increases. However the two-dimensional electron holes are now formed early and field components energy is much higher in particular in the late nonlinear stage of the instability (to compare the left column of Figures 1 and 11). For strongly magnetized plasma (Run 7), the increase of the drift speed of electron beam can excite stronger electrostatic whistler waves, compare with Run 2 . In Figure 12, we can find that the electric field energies $E_{x}^{2}$ and $E_{y}^{2}$ begin to decrease after attaining their maximum values because of waves coalescence. However, from about $\omega_{p e} t=500$, electrostatic whistler waves are excited and the electric field energy in the perpendicular direction $E_{y}^{2}$ increases. In this case, the electron holes begin to form at about $\omega_{p e} t=350$, and they also have mainly one-dimensional structures. From the electric field $E_{x}$ and $E_{y}$ at $\omega_{p e} t=780$, we can find that some regular structures inside the electron holes occur for the corresponding electric field $E_{y}$. Within the holes, a series of islands with alternate positive and negative $E_{y}$ forms as in Run 2. Therefore the parallel cut of $E_{x}$ has bipolar structures while the parallel cut of $E_{y}$ has unipolar structures. From 

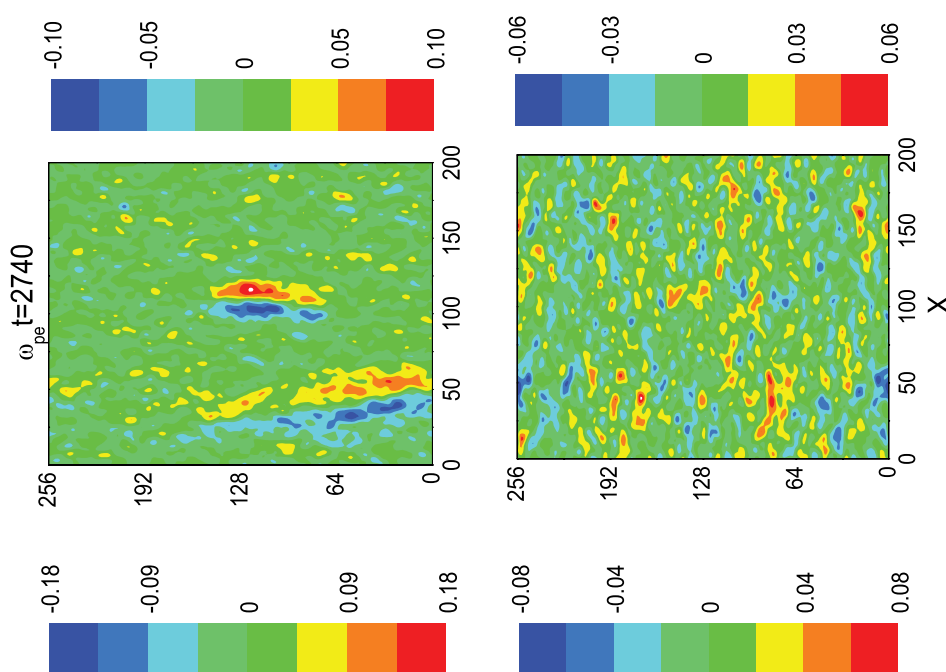

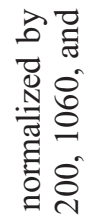
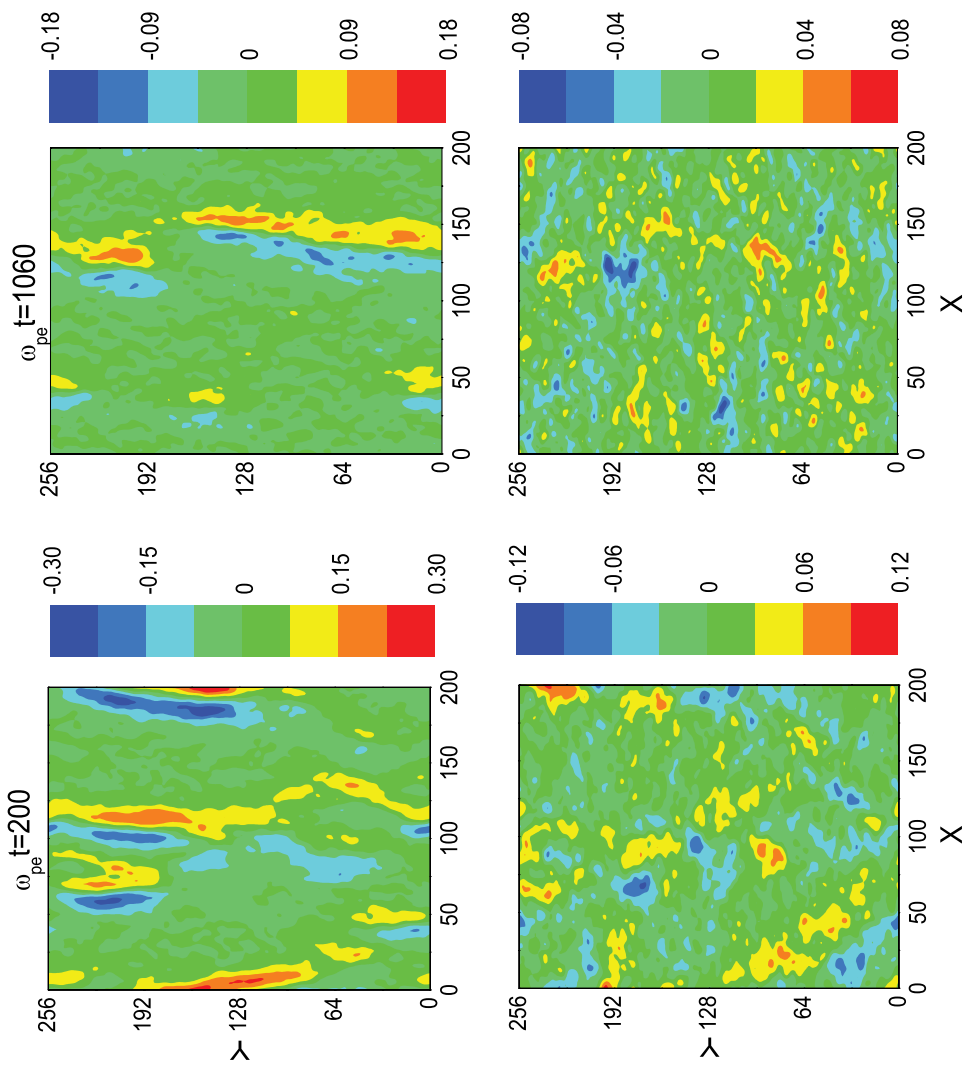

苟

$\frac{1}{3} 3^{\frac{1}{0}}$

सरी

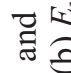

近草

$\cdot \frac{\infty}{0,} \stackrel{0}{3}$

可

융

尊

过

$\approx$

눙

冚䓠

む。

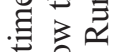

的亭

की

家言令

竞落
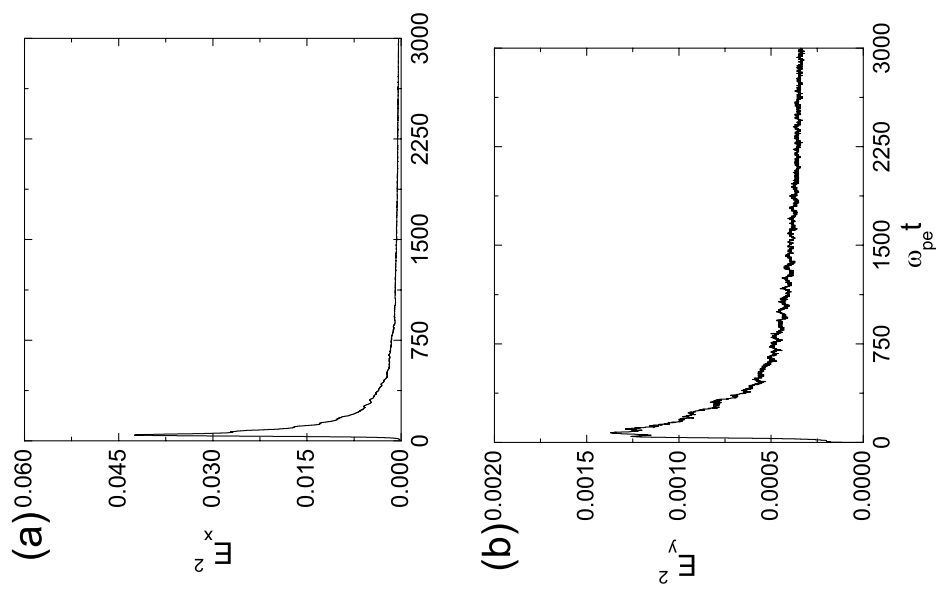

牙

$\because \|$

E.

बำ

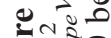

$33^{2}$ ?

证 

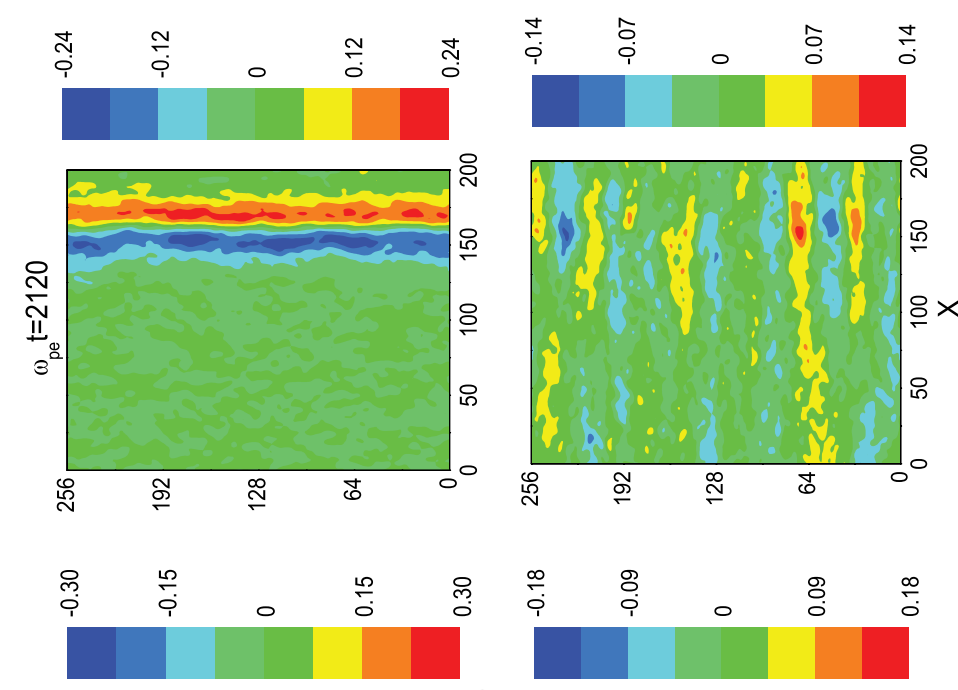

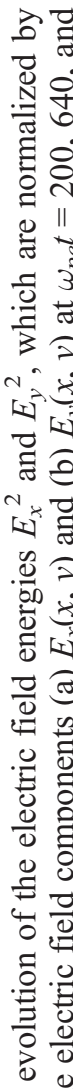
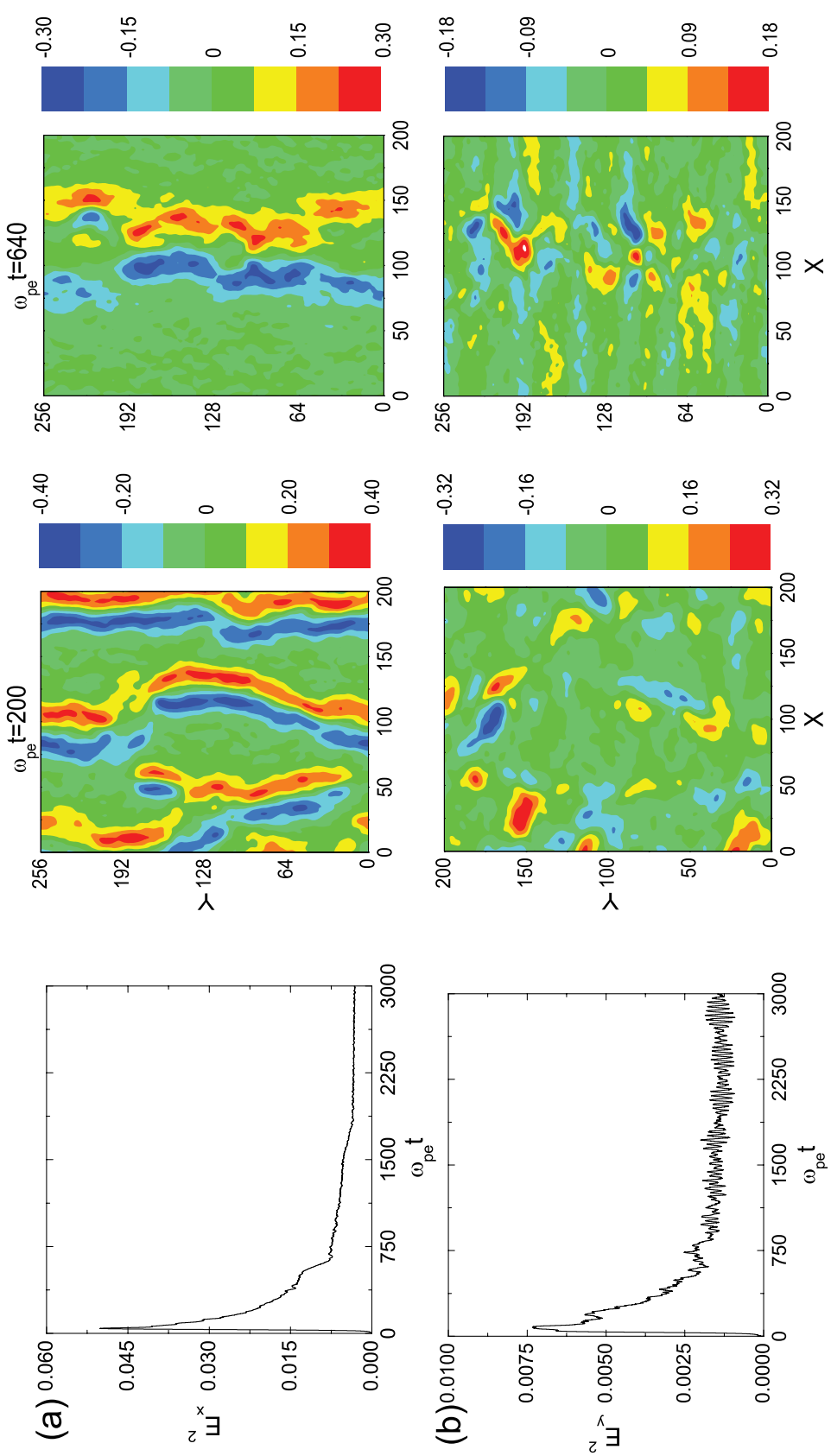

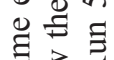

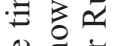

娄

客苜余

के च्र

藏

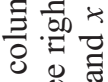

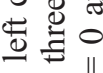

o o

F

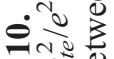

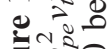

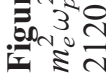



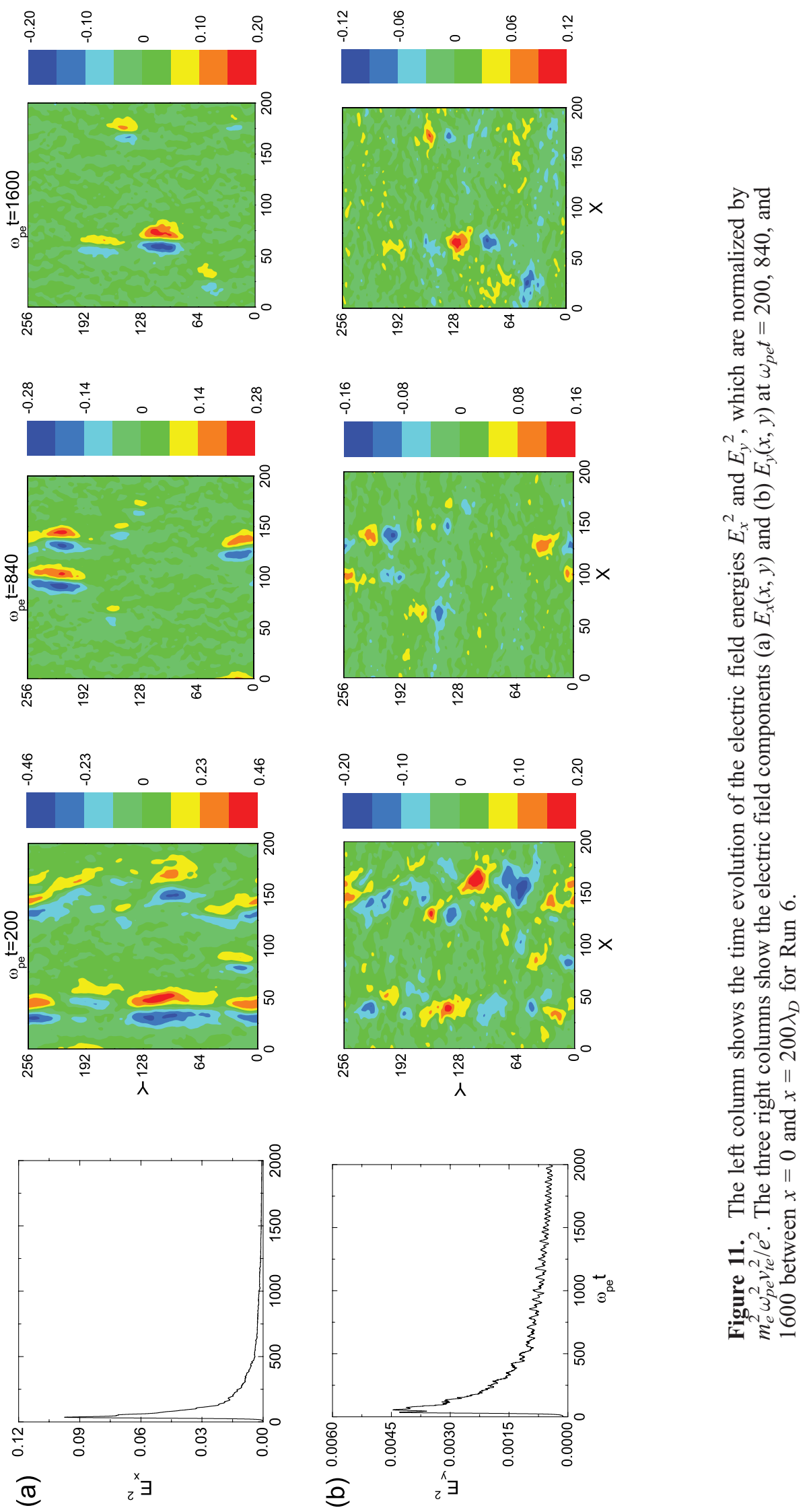

幽䒠

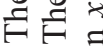

त०

जे

돈 

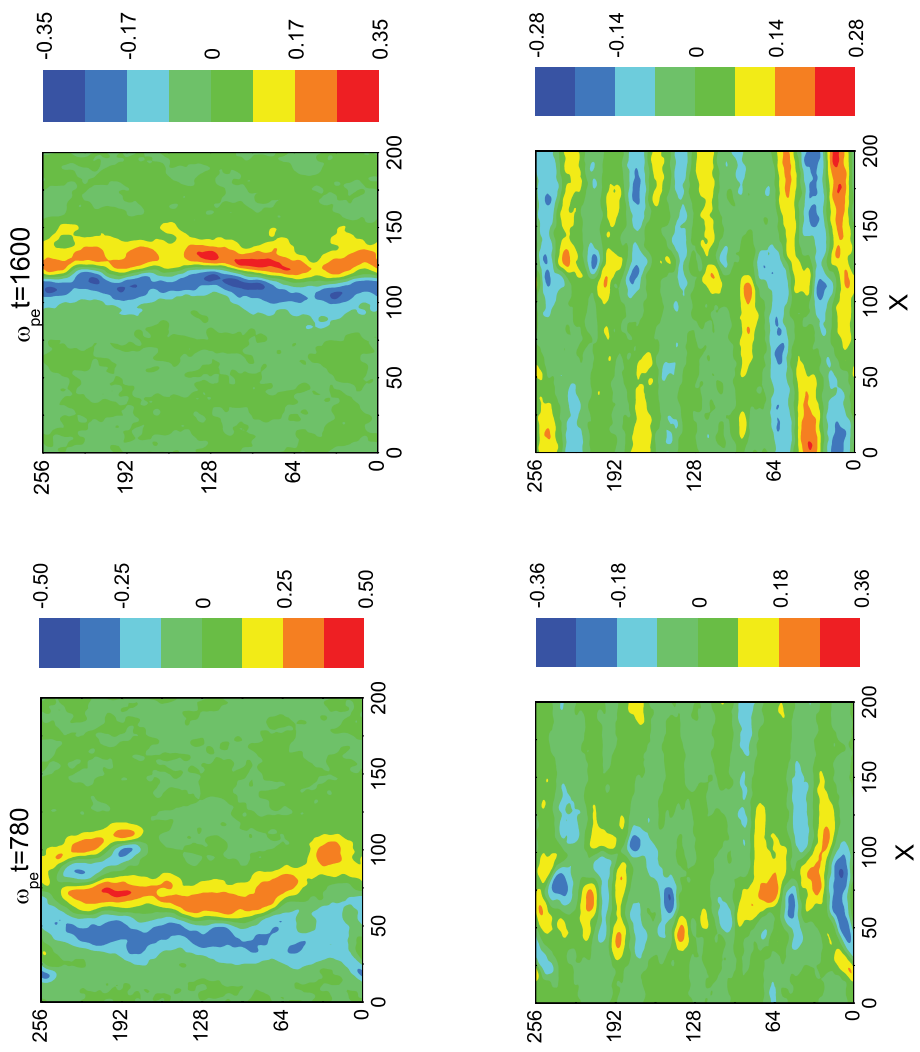

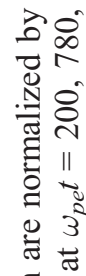

.

N. स

ำ

䒕

ㄴำ

कै

क्षे

ब잉

을 常

葛 晜

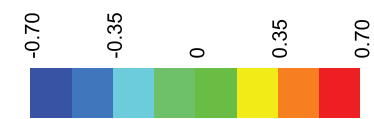

․․

응
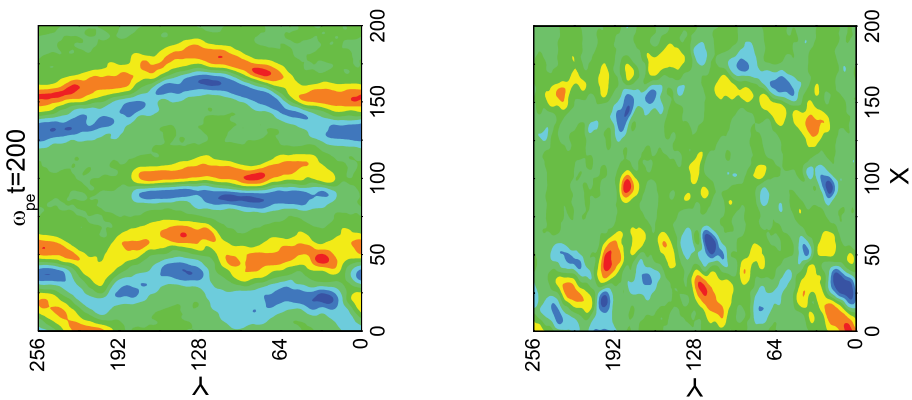

0.

을

更

字志

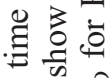

Ð

产 空

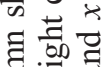

至

잉
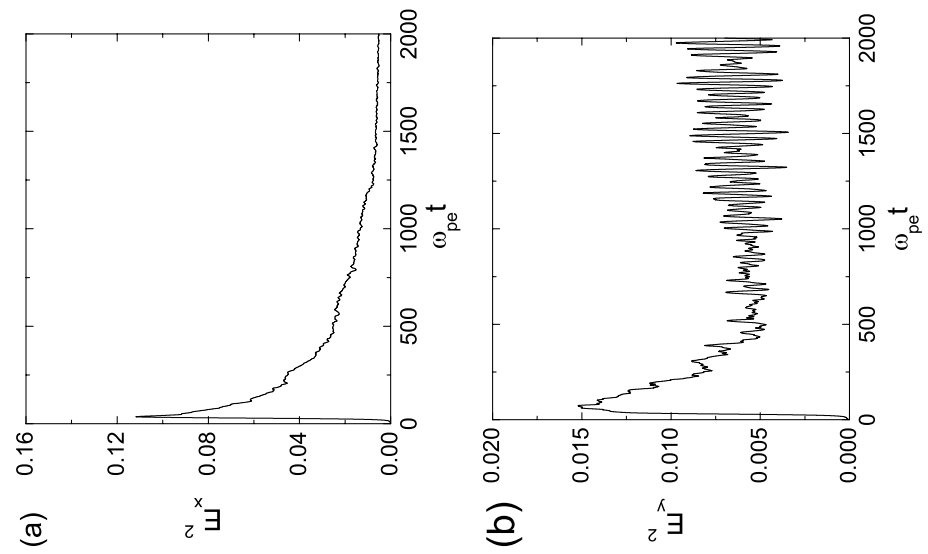

远

을

तथर

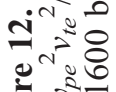

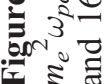

( $\quad{ }_{2}^{x} \exists$ 
about $\omega_{p e} t=900$, the amplitude of the electrostatic whistler waves reaches a noticeable value. As the amplitude of electrostatic whistler waves is sufficiently large, the unipolar $E_{y}$ structures are destroyed. In our simulations, the unipolar $E_{y}$ structures disappear at about $\omega_{p e} t=1200$, and they are replaced by streaked structures as shown by the electric fields $E_{x}$ and $E_{y}$ at $\omega_{p e} t=1600$. Although $E_{x}$ still has obvious bipolar structures, the unipolar structures for the electric field $E_{y}$ disappear. If we still increase the amplitude of the magnetic field, the electrostatic whistler waves can be excited early and stronger. The unipolar structures of $E_{y}$ disappear earlier and they last for shorter time periods (not shown herein).

\subsection{Effects of Ion to Electron Mass Ratio}

[16] Runs 8 and 9 present the evolution of electron bistream instability when ion to electron mass ratio is changed, which are chosen as $\infty$ and 100 in Run 8 and 9, respectively. In this way, we artificially exclude the effects of ion dynamics in Run 9 and amplify the effects of ion dynamics in Run 9. The simulations results are shown in Figures 13 and 14. Compare Run 8 with Run 2, we can find that before the electrostatic whistler waves are excited at about $\omega_{p e} t=1200$, their evolutions are similar. Therefore the unipolar $E_{y}$ structures are obviously unrelated to ion dynamics. In Run 8, the one-dimensional electron holes are formed at about $\omega_{p e} t=300$, and in the holes the parallel cut of $E_{x}$ and $E_{y}$ have bipolar and unipolar structures, respectively (as shown at $\omega_{p e} t=640$ ). When the electrostatic whistler waves are excited, they are much stronger than that in Run 2, and the bipolar $E_{y}$ structures are destroyed at about $\omega_{p e} t=1500$ and streaked structures are formed. In Run 9, before the low hybrid waves are excited at about $\omega_{p e} t$ $=250$, its evolution is similar to Run 2 . However, after the low hybrid waves are excited, they can modulate the electron holes. The parallel cut of $E_{x}$ and $E_{y}$ have bipolar and unipolar structures, respectively, as described Miyake et al. [2000] and Umeda et al. [2002]. With the increase of the amplitude of the low hybrid waves, the electron holes are broken into several 2D holes as shown at $\omega_{p e} t=640$. At about $\omega_{p e} t=1440$, a streaked structure is formed. As discussed by Oppenheim et al. [2001], ions only become important when the angle between the background magnetic field and wave vector is larger than $\pi / 2-\sqrt{m_{i} / m_{e}}$. Therefore, if the ion to electron ratio is artificially decreases, the low hybrid waves may be excited and modulate the electron holes.

\section{Discussion and Conclusions}

[17] In this paper, by performing 2D electrostatic particlein-cell simulations we investigate the nonlinear evolution of bi-stream instability, with emphasis on the unipolar structures of $E_{\perp}$ in electron holes. Our results show that during the nonlinear stage of the bi-stream instability the electron holes can be formed through wave coalescence. In the weakly magnetized plasma, the electron holes have twodimensional structures, which are isolated in both the parallel and perpendicular directions. Within such holes, the parallel cut of $E_{y}$ have unipolar structures. In strongly magnetized plasma, electron holes have mainly onedimensional structures elongated along the $y$ direction, where a series of islands (with alternate positive and negative $E_{y}$ ) develop along the direction perpendicular to the background magnetic field. Again, the parallel cut of $E_{y}$ has unipolar structures at the location of the holes.

[18] Our results show that the generation mechanism of the unipolar structure of $E_{y}$ in the parallel cut of the electron holes is not due to ion dynamics. This is demonstrated by comparing two cases, where realistic ion to electron mass ratio $m_{i} / m_{e}=1836$ and immobile ions are used. Their evolutions are similar before the electrostatic whistler waves are excited, and the unipolar $E_{y}$ structures are both recovered. Rather, these structures are attributed to the balance of transverse instability (which tends to destroy electron holes) and the stabilization of the relatively strong magnetic field (which tends to stabilized electron holes) [Muschietti et al., 2000]. As the strength of the background magnetic field is large enough, the electron holes have mainly one-dimensional structures. The trapped electrons in electron holes bounce in the parallel direction, at the same time they gyrate in the background magnetic field. As a consequence, the charge density varies along the perpendicular direction inside electron holes, which are responsible for the unipolar $E_{y}$ structures. In the weakly magnetized plasma $\left(\omega_{b}<\Omega_{e}<\right.$ $\omega_{p e}$, and $\Omega_{e}$ is comparable to $\omega_{p e}$, where $\omega_{b}$ is the bounce frequency of electron trapped in electron holes), the balance between the transverse instability and stabilization of the magnetic field leads to the formation of two-dimensional electron holes. When the background magnetic field continues to decrease and becomes sufficiently weak $\left(\Omega_{e}<\right.$ $\left.\omega_{p e}\right)$, the transverse instability dominates, and the electron holes are unstable to transverse instability. Then, no electron holes can be formed.

[19] The two-dimensional electron holes in weakly magnetized plasma have also been studied by Umeda et al. [2006]. Our simulations show that the increase of the electron perpendicular temperature can reduce the transverse instability and stabilize the electron holes, as indicated by Muschietti et al. [2000]. One-dimensional electron holes may be formed during the evolution of bi-steam instability when the electron temperature anisotropy is sufficiently large. In strongly magnetized plasma, the parallel cut of $E_{y}$ has unipolar structures within electron holes, which are implied by Goldman et al. [1999] and Oppenheim et al. [1999, 2001]. In this paper, we discuss the generation mechanism of such structures, which are attributed to the variations of the charge density along the perpendicular direction. Such structures of $E_{y}$ can last for hundreds to thousands of electron plasma periods until they are destroyed by the electrostatic whistler waves. The increase of either the drift speed of the electron beam or the background magnetic field can increase the amplitude of electrostatic whistler waves, and destroys the unipolar structures of $E_{\perp}$ easier and earlier, which at last forms streaked structures in the whole simulation domain. Another thing we should point out is that we are interesting in the unipolar structures of $E_{\perp}$ in the electron holes before the electrostatic whistler waves have sufficiently large amplitude. When the electrostatic whistler waves are sufficiently strong, the one-dimensional electron holes may also be broken into two-dimensional after several thousand electron plasma periods as discussed by Oppenheim et al. and Umeda et al. [2006]. However such two-dimensional electron holes 

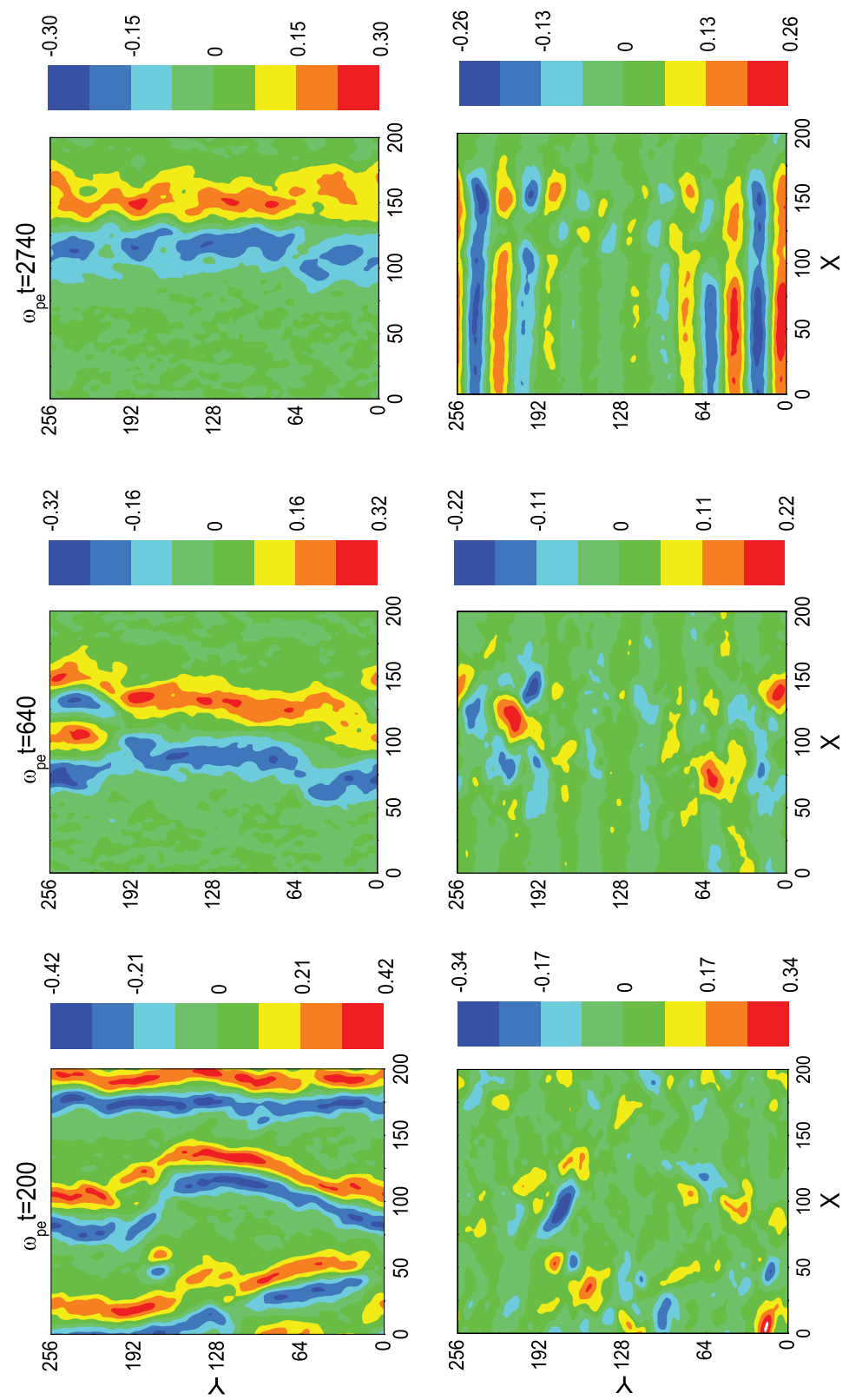

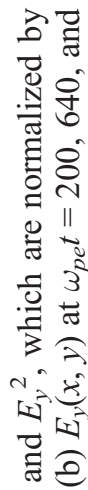

过茎

.03

क्षे

흉

号

苍 응

인웡

总兽

$\mathscr{g}$

录合

둔

站管余

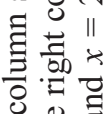
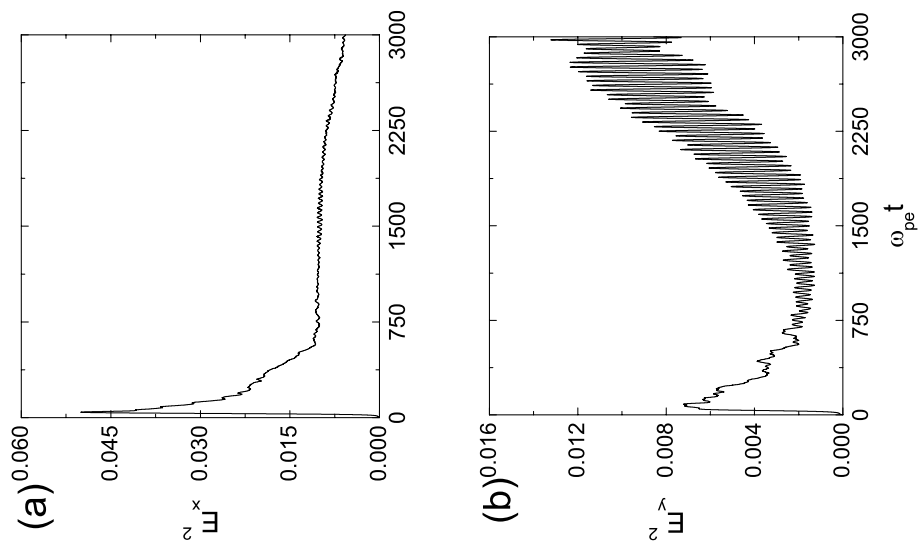

屯ํㅣㅇ

E

त.

$9 \frac{0}{2}$

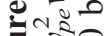

ำ 

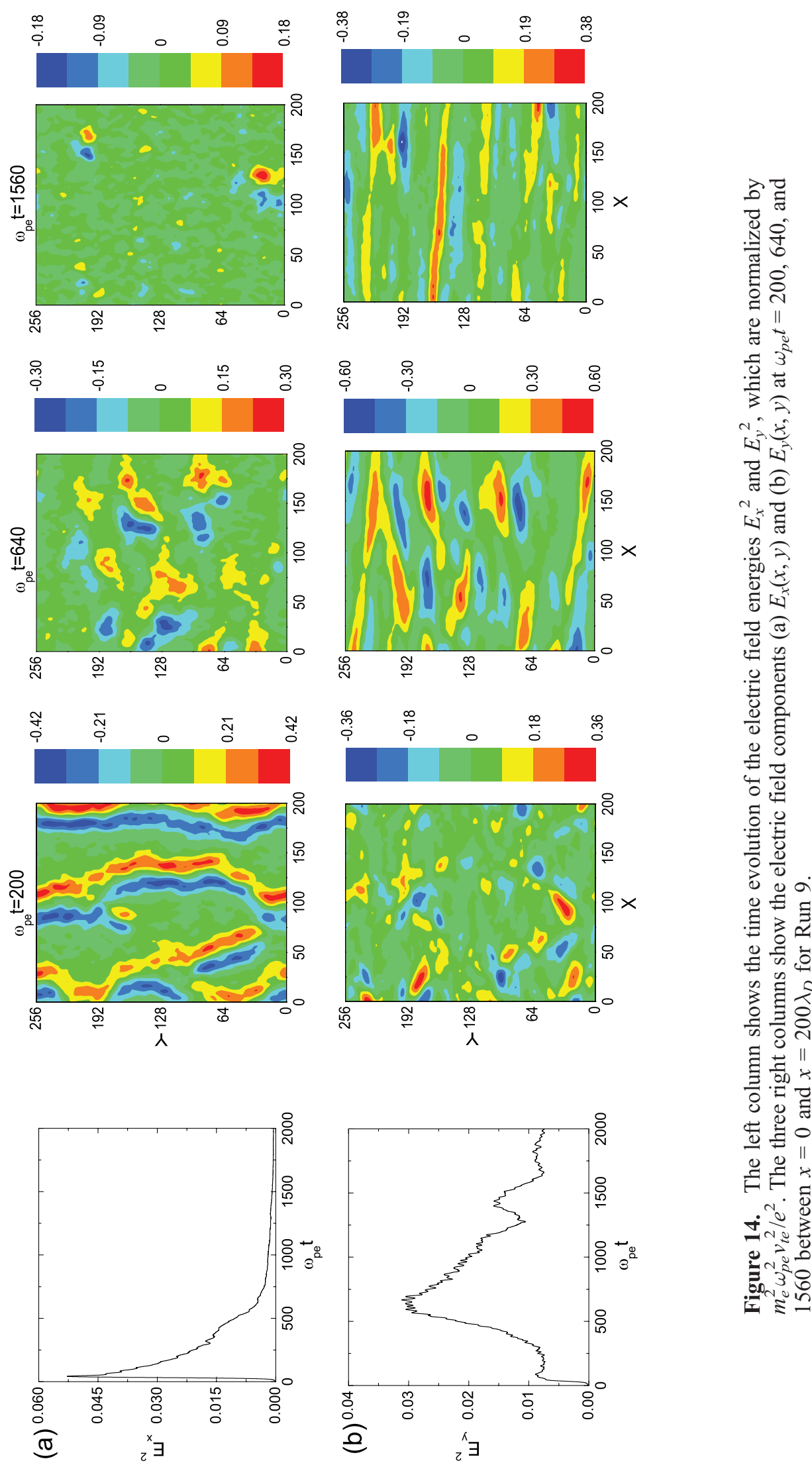

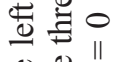

急事

ㄱํㄹ

$2 \pi{ }^{2}=0$

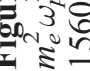


are different from those formed in weakly magnetized plasma. Here, the perpendicular electric field forms streaked structures in the whole simulation domain, and the parallel cut of $E_{\perp}$ has no unipolar structures in electron holes.

[20] On the experiment view point, the unipolar structures of the parallel cut of the perpendicular electric field have been observed by Polar and Fast spacecraft in the auroral region [Ergun et al., 1998a, 1998b; Franz et al., 1998, 2005; Grabbe and Menietti, 2006]. Our present simulations showed that the perpendicular electric field $\left(E_{y}\right)$ has regular structures (islands), which develop inside the one-dimensional electron holes along the perpendicular direction. Simultaneously, a parallel cut of the perpendicular electric field $\left(E_{y}\right)$ has unipolar structures. The generation mechanism of such structure is governed by electron dynamics, which force trapped electrons to accumulate locally in electron holes. The structures are shown to last for long time provided that the drift velocity is not too large (If we can assume the $E_{\perp}$ structures can last one thousand plasma periods as described in our paper, then their lifetime is about several $\mathrm{ms}$ in the auroral region). These results give a possible explanation of the unipolar structures of the parallel cut of $E_{\perp}$ observed in the auroral region, where plasma is strongly magnetized. Of course, our simulations use 2D geometry with periodic boundary condition in the $y$ direction. Such periodic boundary condition assumes that the 1D electron holes have infinite length in the $y$ direction and the edge effect is neglected, while the 2D geometry neglects the transverse instability in the $z$ direction. However, in reality, the electron holes are three-dimensional, and like tubes [Ng and Bhattacharjee, 2005; Ng et al., 2006]. Therefore the comparison between our simulations and observation data, as well as 3D BGK mode theory, is only qualitatively. 3D PIC simulations of electron bi-stream instability are our future investigation.

[21] Acknowledgments. This research was supported by the National Science Foundation of China (NSFC) under grants 40725013, 40674093, and the Chinese Academy of Sciences grant KZCX3-SW-144.

[22] Amitava Bhattacharjee thanks the reviewers for their assistance in evaluating this paper.

\section{References}

Bale, S. D., P. J. Kellogg, D. E. Larson, R. P. Lin, K. Goetz, and R. P. Lepping (1998), Bipolar electrostatic structures in the shock transition region: Evidence of electron phase holes, Geophys. Res. Lett., 25, 2929-2932.

Bernstein, I. B., J. M. Greene, and M. D. Kruskal (1957), Exact nonlinear plasma oscillations, Phys. Rev., 108, 546-550.

Berthomier, M., L. Muschietti, J. W. Bonnell, I. Roth, and C. W. Carlson (2002), Interaction between electrostatic whistlers and electron holes in the auroral region, J. Geophys. Res., 107(A12), 1463, doi:10.1029/ 2002JA009303.

Cattell, C., J. Crumley, J. Dombeck, J. Wygant, and F. S. Mozer (2002), Polar observations of solitary waves at the Earth's magnetopause, Geophys. Res. Lett., 29(5), 1065, doi:10.1029/2001GL014046.

Chen, L. J., J. Pickett, P. Kintner, J. Franz, and D. Gurnett (2005), On the width-amplitude inequality of electron phase space holes, J. Geophys. Res., 110, A09211, doi:10.1029/2005JA011087.

Decyk, V. K. (1995), Skeleton PIC codes for parallel computers, Comput. Phys. Commun., 87, 87-94.

Ergun, R. E., C. W. Carlson, J. P. McFadden, F. S. Mozer, L. Muschietti, I. Roth, and R. J. Strangeway (1998a), Debye-scale plasma structures associated with magnetic-field-aligned electric fields, Phys. Rev. Lett., $81,826-829$.

Ergun, R. E., et al. (1998b), Fast satellite observations of large-amplitude solitary structures, Geophys. Res. Lett., 25, 2041-2044.

Franz, J. R., P. M. Kintner, and J. S. Pickett (1998), POLAR observations of coherent electric field structures, Geophys. Res. Lett., 25, 1277-1280.
Franz, J. R., P. M. Kintner, C. E. Seyler, J. S. Pickett, and J. D. Scudder (2000), On the perpendicular scale of electron phase-space holes, Geophys. Res. Lett., 27, 169-172.

Franz, J. R., P. M. Kintner, J. S. Pickett, and L. J. Chen (2005), Properties of small-amplitude electron phase-space holes observed by Polar, J. Geophys. Res., 110, A09212, doi:10.1029/2005JA011095.

Goldman, M. V., M. M. Oppenheim, and D. L. Newman (1999), Nonlinear two-stream instabilities as an explanation for auroral bipolar wave structures, Geophys. Res. Lett., 26, 1821-1824.

Grabbe, C. L., and J. D. Menietti (2006), Broadband electrostatic wave observations in the auroral region on Polar and comparisons with theory, J. Geophys. Res., 111, A10226, doi:10.1029/2006JA011602.

Krasovsky, V. L., H. Matsumoto, and Y. Omura (1997), Bernstein-GreenKruskal analysis of electrostatic solitary waves observed with Geotail, J. Geophys. Res., 102, 22,131-22,139.

Lu, Q. M., and D. S. Cai (2001), Implementation of parallel plasma particle-in-cell codes on PC cluster, Comput. Phys. Commun., 135, 93-104. Lu, Q. M., D. Y. Wang, and S. Wang (2005a), Generation mechanism of electrostatic solitary waves in the Earth's auroral region, J. Geophys. Res., 110, A03223, doi:10.1029/2004JA010739.

Lu, Q. M., S. Wang, and S. Wang (2005b), Electrostatic waves in an electron-beam plasma system, Phys. Plasmas, 12, 072903.

Mangeney, A., C. Salem, C. Lacombe, J. L. Bougeret, C. Perche, R. Manning, P. J. Kellogg, K. Goetz, S. J. Monson, and J. M. Bosqued (1999), Ann. Geophys., 17, 307-320

Matsumoto, H., H. Kojima, T. Miyatake, Y. Omura, M. Okada, I. Nagano, and M. Tsutsui (1994), Electrostatic solitary waves (ESW) in the magnetotail: BEN wave forms observed by Geotail, Geophys. Res. Lett., 21, $2915-2918$

Miyake, T., Y. Omura, and H. Matsumoto (2000), Electrostatic particle simulations of solitary waves in the auroral region, J. Geophys. Res., $105,23,239-23,249$

Mottez, F., S. Perraut, A. Roux, and P. Louarn (1997), Coherent structures in the magnetotail triggered by counterstreaming electron beams, J. Geophys. Res., 102, 11,399-11,408.

Muschietti, L., R. E. Ergun, I. Roth, and C. W. Carlson (1999), Phase-space electron holes along magnetic field lines, Geophys. Res. Lett., 26, $1039-$ 1096.

Muschietti, L., I. Roth, C. W. Carlson, and R. E. Ergun (2000), Transverse instability of magnetized electron holes, Phys. Rev. Lett., 85, 94-97.

Newman, D. L., M. V. Goldman, M. Spector, and F. Perez (2001), Dynamics and instability of electron phase-space tubes, Phys. Rev. Lett., $86,1239-1242$

Ng, C. S., and A. Bhattacharjee (2005), Bernstein-Greene-Kruskal modes in a three-dimensional plasma, Phys. Rev., 95, 245004.

Ng, C. S., A. Bhattacharjee, and F. Skiff (2006), Weakly collisional Landau damping and three-dimensional Bernstein-Greene-Kruskal modes: New results on old problems, Phys. Plasmas, 13, 055903.

Omura, Y., H. Kojama, and H. Matsumoto (1994), Computer simulation of electrostatic solitary waves: A nonlinear model of broadband electrostatic noise, Geophys. Res. Lett., 21, 2923-2926.

Omura, Y., H. Matsumoto, T. Miyake, and H. Kojima (1996), Electron beam instabilities as generation mechanism of electrostatic solitary waves in the magnetotail, J. Geophys. Res., 101, 2685-2697.

Oppenheim, M., D. L. Newman, and M. V. Goldman (1999), Evolution of electron phase-space holes in a 2D magnetized plasma, Phys. Rev. Lett., $83,2344-2347$

Oppenheim, M. D., G. Vetoulis, D. L. Newman, and M. V. Goldman (2001), Evolution of electron phase-space holes in 3D, Geophys. Res. Lett., 28, 1891-1894.

Pickett, J. S., L. J. Chen, S. W. Kahler, O. Santolik, D. A. Gurnett, B. T. Tsurutani, and A. Balogh (2004), Isolated electrostatic structures observed through the Cluster orbit: Relation to magnetic field strength, Ann. Geophys., 22, 2515-2523.

Umeda, T., Y. Omura, H. Matsumoto, and H. Usui (2002), Formation of electrostatic solitary waves in plasmas: Particle simulations with open boundary conditions, J. Geophys. Res., 107(A12), 1449, doi:10.1029/ 2001JA000286.

Umeda, T., Y. Omura, T. Miyaka, H. Matsumoto, and M. Ashour-Aballa (2006), Nonlinear evolution of the electron two-stream instability: Twodimensional particle simulations, J. Geophys. Res., 111, A10206, doi:10.1029/2006JA011762.

B. Lembege, Centre d'étude des Environnements Terrestre et Planétaires, CNRS Université de Versailles-Saint Quentin, Vélizy 78140, France.

Q. M. Lu, J. B. Tao, and S. Wang, CAS Key Laboratory of Basic Plasma Physics, School of Earth and Space Sciences, University of Science and Technology of China, Hefei, Anhui 230026, China. (qmlu@ustc.edu.cn) 